DIVISION OF THE HUMANITIES AND SOCIAL SCIENCES

CALIFORNIA INSTITUTE OF TECHNOLOGY

PASADENA, CALIFORNIA 91125

RISK, AMBIGUITY, AND THE SEPARATION OF UTILITY AND BELIEFS

Paolo Ghirardato

California Institute of Technology

Massimo Marinacci

Università di Torino

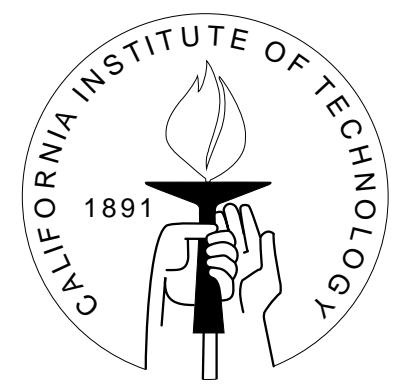

SOCIAL SCIENCE WORKING PAPER 1085

March 2000 


\title{
Risk, Ambiguity, and the Separation of Utility and Beliefs
}

\author{
Paolo Ghirardato Massimo Marinacci
}

\begin{abstract}
We introduce and characterize axiomatically a general model of static choice under uncertainty, which is possibly the weakest model in which a separation of cardinal utility and a representation of beliefs is achieved. Most of the popular non-expected utility models in the literature are special cases of it.

To prove its usefulness, we show that the model can be used to generalize several well known results on the characterization of risk aversion. Elsewhere [15] we have shown that it can be fruitfully applied to the problem of characterizing a notion of ambiguity aversion, as the separation of utility and beliefs that we achieve can be used to identify and remove aspects of risk attitude from the decision maker's behavior.
\end{abstract}

JEL classification numbers: D81

Key words: Risk Aversion, Ambiguity Aversion, Biseparable Preferences, Choquet Expected Utility, Maxmin Expected Utility, Probabilistic Beliefs 


\title{
Risk, Ambiguity, and the Separation of Utility and Beliefs*
}

\author{
Paolo Ghirardato $\quad$ Massimo Marinacci
}

\section{Introduction}

In this paper, we introduce and characterize axiomatically a general model of static choice under uncertainty, whose main interest lies in being the weakest known model in which the separation between cardinal utility and a representation of beliefs is achieved. Though very general, the model has enough structure to prove some interesting results. We illustrate this by showing some characterizations of risk aversion. In a companion paper [15], we show how the model can also be used to provide a very general characterization of a notion of ambiguity aversion.

The motivation for looking at models of choice under uncertainty that generalize the classical subjective expected utility (SEU) model of Savage [27] and Anscombe and Aumann [2] is well known. There is a wealth of evidence as to the descriptive limitations of the SEU model. The two most popular problems are the so-called Allais paradox, first observed by Allais [1], and the so-called Ellsberg paradox, first observed by Ellsberg [12]. Besides showing that the strong separability enjoyed by expected utility is not descriptively accurate, these 'paradoxes' raise the issue of whether it is normatively compelling. A large number of extensions of SEU have been developed that weaken separability and rationalize some of these violations. There are models that rationalize the 'ambiguity averse' behavior of the Ellsberg paradox, like the Choquet expected utility (CEU) model of Schmeidler [28], or the maxmin expected utility (MEU) model of Gilboa and Schmeidler [18]. ${ }^{1}$ There are models that rationalize the choices in the Allais paradox, like the subjective rank-dependent expected utility (RDEU) model (based on Quiggin [25] and Yaari [32]), or the more general model of probabilistically sophisticated (PS) preferences

* We are grateful to Kim Border, Peter Wakker and especially Fabio Maccheroni for helpful comments and discussion. This paper was mostly written while Marinacci was with the Università di Bologna. Marinacci gratefully acknowledges the financial support of MURST. Ghirardato's E-mail: paolo@hss.caltech.edu; URL: www.hss.caltech.edu/ paolo/ghiro.html. Marinacci's address: Dipartimento di Statistica e Matematica Applicata, Università di Torino, Piazza Arbarello 8, 10122 Torino, ITALY; E-mail: massimo@econ.unito.it; URL: ww. dse.unibo.it/marinacc/page1.htm.

${ }^{1}$ See Appendix A for references to axiomatizations of CEU and MEU in a subjective framework $\grave{a}$ la Savage. 
of Machina and Schmeidler [21]. Finally, there are models that rationalize additional features of observed behavior, like the cumulative prospect theory (CPT) of Tversky and Kahneman [29].

Though most of these models share some features, such are the differences between them that so far very few papers have attempted to find which nontrivial results can be proved for most, if not all, of them. Doing so requires finding a 'common denominator' model with very little structure, yet enough structure for defining some key mathematical aspects of the representation, and proving results about them. Here, we present one such model, called the 'biseparable preferences' model, that generalizes almost all the models mentioned above. A preference relation is biseparable (short for 'binary separable') if it can be represented by a functional $V$ which is monotonic with respect to state-by-state dominance, and which on binary acts has an 'expected utility' form, but with respect to a not necessarily additive measure. Precisely, if we denote by $u$ the restriction of $V$ to constant acts, there is a capacity (a monotonic set-function) $\rho$ such that

$$
V(f)=u(x) \rho(A)+u(y)(1-\rho(A)),
$$

where $f$ is the act which pays $x$ if event $A$ obtains and $y$ otherwise, with $x$ preferred to $y$. Under standard continuity assumptions, such $V$ is cardinal whenever there is at least an event with nontrivial $\rho$ weight. This implies that $u$ is cardinal, and that $\rho$ is unique. Thus, a decision maker (DM) whose preferences are biseparable evaluates consequences by a cardinal state-independent utility index, and evaluates bets by a unique capacity, that we call his willingness to bet. No restrictions (beyond monotonicity) are imposed on how the DM evaluates the more complex nonbinary acts. This is why the biseparable preferences model encompasses all the models listed above, with the exception of the PS and CPT models, which are anyway not more general than it (see the discussion below): As observed earlier, we are not aware of any other model which is more general and yields a separation of the DM's cardinal utility index from the unique representation of his willingness to bet. ${ }^{2}$

Using the special horse races-lottery wheels setting of the Anscombe-Aumann paper [2], we also provide a variant of the biseparable preference model in which the DM's utility function is linear on lotteries. We call this variant the c-linear biseparable preference model. To the best of our knowledge, it provides the weakest model which separates willingness to bet from a cardinal and affine utility function.

Thanks to the cardinality of utility, biseparable preferences provide a general model to which we are able to extend several results on the characterization of risk aversion for SEU preferences: (1) a natural 'more risk averse than' relation between preferences is characterized by the existence of a concave transformation between utilities; (2) the classical notion of risk aversion as preference for the expected value (duly transposed to a purely subjective framework) is characterized by concavity of the utility; (3) a notion

2 In general, there may be multiple representations of the DM's 'likelihood ordering' over bets, but the capacity which satisfies Eq. (1) is unique. 
of preference for diversification in the choice among bets is again characterized by the concavity of utility.

However, some care has to be taken in the interpretation of these results. Since we are in an environment of pure uncertainty, and since biseparable preferences have very little structure, the notion of 'risk aversion' needs definition. The most popular definition of risk aversion for non-SEU preferences is aversion to mean-preserving spreads. Its interpretation hinges crucially on the existence of a sufficiently rich set of events on which the DM has probabilistic beliefs, and on the assumption that the DM only cares about the 'distribution' that an act induces on the set of consequences (part of what Machina and Schmeidler term 'probabilistic sophistication'). Absent those preconditions, aversion to mean-preserving spreads is meaningless. A more general definition of risk aversion (based on Yaari [31]) is the following: define a comparative relation among preferences to be interpreted as 'more risk averse than', conventionally define a set of preferences to represent 'risk neutrality', and then call 'risk averse' any preferences which is more risk averse than a risk neutral one. This is quite compelling, but as we observed in [15], the comparative relation has to be carefully devised if we are to distinguish risk aversion from other behavioral features, like ambiguity aversion. In particular, we need to avoid confusing differences in beliefs with differences in risk aversion. In the SEU framework, this is easily solved by assuming that the preferences which are compared have identical beliefs (possibly because they reflect an 'objective' probability). When dealing with a model as general as biseparable preferences, this is not as straightforward: What do we mean by 'same beliefs'? Here we take the simple route of comparing preferences with the same willingness to bet. Some readers may be concerned that the willingness to bet contains information on the risk attitude of the DM that we are thus omitting from consideration. For this reason, we prefer to say that what is characterized in our results is just an aspect of the DM's risk attitude, that we call the DM's cardinal risk attitude. Intuitively, cardinal risk attitude is what explains a DM's willingness to trade off the payoff in case of a win with the tradeoff in case of a loss in bets on some fixed event, which for SEU preferences is characterized by the behavior of the utility function. ${ }^{3}$ Intuitively, we attribute such behavior to the DM's risk attitude because we think that it cannot provide us any information as to the ambiguity the DM perceives about that event, and how he reacts to such ambiguity.

As observed earlier, another example of the potential of the biseparable preferences model is our study of ambiguity and ambiguity attitudes in [15]. In that paper we propose a notion of ambiguity aversion and then notions of ambiguity for acts and events. We then provide their characterizations for any biseparable preference. For instance, we show that if a biseparable preference is ambiguity averse in the sense we propose, then its willingness to bet $\rho$ is dominated by a probability, and also that the set of events which are unambiguous for an ambiguity averse (or loving) biseparable preference is the collection of all the $A^{\prime}$ 's such that $\rho(A)+\rho\left(A^{c}\right)=1$. Biseparable preferences play a key role in this analysis because they provide the most general model for which it is possible

3 In saying a bet 'on' an event, we imply that a better payoff ('win') is the result of the event obtaining. 
to cleanly separate cardinal risk and ambiguity attitudes. ${ }^{4}$

Besides their generality and potential, ${ }^{5}$ biseparable preferences can be useful in obtaining a new (non-SEU) choice-theoretic derivation of subjective probability, which is quite different from the one presented by Machina and Schmeidler [21]. In fact, we show that a simple and natural reinforcement of the axioms that characterize biseparable preferences yields a DM with probabilistic beliefs: His willingness to bet $\rho$ is a probability measure (i.e., it is additive). Such DM is not necessarily a SEU maximizer, and may not even be 'probabilistically sophisticated' in the sense of Machina and Schmeidler. Thus, the class of preferences with probabilistic beliefs that we characterize is different from the class of PS preferences (clearly, with a common element given by SEU preferences). The model we present does not necessarily feature what many consider a natural rationality requirement, but it has the advantage of allowing results like those on cardinal risk aversion described earlier.

\section{The Related Literature}

In terms of the preference model, there are few papers that offer a model as general as the biseparable preference model. As mentioned earlier, Machina and Schmeidler [21] and Tversky and Kahneman [29] provide alternative models which are not encompassed by it.

The PS model has different scope from the biseparable preference model: The two models really embody different rationality restricitions on preferences. ${ }^{6}$ They are somewhat symmetric in their final objective: Machina and Schmeidler are interested in obtaining a unique probabilistic belief which is used in a PS fashion, so as to provide a fully subjective foundation to all the non-EU models of choice under risk. In contrast, our objective is obtaining a cardinal utility, so that cardinal risk and ambiguity attitude can be clearly separated. For this reason, the PS model provides a little structure on the 'functional' representation of preferences over all acts, whereas we are very precise on the functional representation, but only over the binary acts. Because of its functional generality, the results on the characterization of risk aversion that one can obtain with PS preferences are limited.

On the other hand, CPT is a model with a very specific functional structure. The main reason why the preferences they describe are not biseparable is that they establish

\footnotetext{
${ }^{4}$ The reader who thinks that risk aversion is not fully captured by cardinal risk aversion should be warned that the notion of ambiguity aversion in [15] comprises also the mentioned residual aspects of risk aversion.

${ }^{5}$ Other applications of biseparability are the following: In [16], we show that a seemingly harmless technical condition (range convexity of the willingness to bet) can force ambiguity averse biseparable preferences to have probabilistic beliefs. Ozdenoren [23] shows that some results on auctions that he proves for MEU preferences can be extended to biseparable preferences.

${ }^{6}$ However, it should be remarked that the intersection between biseparable preferences and probabilistically sophisticated preferences is nontrivial, including for instance the RDEU preferences.
} 
a reference point, and allow the DM's willingness to bet to be different depending on whether he considers bets with 'gains' (consequences preferred to the reference point), or bets with 'losses' (consequences to which the reference point is preferred). It would be straightforward to generalize the model we present to allow such behavior, thus obtaining what could be called the cumulative biseparable preferences model. Needless to say, all the results we prove here are true if we focus our attention to gains or losses only (so that CPT collapses to CEU).

Two other axiomatic papers that are close to this paper are Casadesus-Masanell, Klibanoff and Ozdenoren [4] and Nakamura [22]. As a technical lemma in their axiomatic characterizations of respectively MEU and CEU preferences both papers present a biseparable representation of preferences like that in Eq. (1). However, their representation holds only for bets on a specific event $A$, rather than for bets on any event.

Finally, there are some papers which address the characterization of behavioral features, like risk aversion, for large classes of preferences. ${ }^{7}$ In his seminal paper on risk aversion [31], Yaari presents a comparatively based notion of risk aversion (like the one described earlier), and provides a characterization for a very general class of preferences. However his characterization requires differentiability assumptions that are not easy to characterize behaviorally. Thus, his result is quite different from our result on cardinal risk aversion that we listed earlier as (1). Epstein [13] also uses a comparative approach to define ambiguity aversion, obtaining a notion which is quite different from the one in our [15] (which contains a detailed comparison of the two notions). He provides a characterization of his notion for CEU, MEU and a class of preferences which satisfies an eventwise differentiability condition. He does not address the characterization of risk aversion for any of these models.

\section{Organization}

The paper is organized as follows. Section 1 introduces some required notation and terminology. Section 2 introduces formally the class of the biseparable and c-linear biseparable preferences, it shows some properties that they possess, and provides some examples. Section 3 contains the results on cardinal risk aversion. Section 4 provides the axiomatizations of the two preference models, and shows how to obtain probabilistic beliefs. Section 5 closes by looking at a smaller class of preferences which satisfies a natural invariance property. The appendices contain some additional definitions (App. A), a technical remark (App. B), and the proofs for all the results in the paper (App. C).

7 Clearly, many other papers have tackled the problem of characterizing behavioral features in the context of specific decision models. Here we only focus on those which deal with several different models. 


\section{Set-Up and Preliminaries}

The Savage-style setting we use consists of a set $S$ of states of the world, an algebra $\Sigma$ of subsets of $S$ called events, and a set $X$ of consequences. We denote by $\mathcal{F}$ the set of all the simple acts: finite-valued functions $f: S \rightarrow X$ which are measurable with respect to $\Sigma$. In contrast, let $B(\Sigma)$ denote the set of all real-valued $\Sigma$-measurable simple functions. We say that a functional $I: B(\Sigma) \rightarrow \mathbb{R}$ is monotone if $\phi \geq \psi$ implies $I(\phi) \geq I(\psi)$ for all $\phi, \psi \in B(\Sigma)$.

For $x \in X$ we define $x \in \mathcal{F}$ to be the constant act $x(s)=x$ for all $s \in S$. So, with the usual slight abuse of notation, we identify $X$ with the subset of the constant acts in $\mathcal{F}$. Moreover, $x A y$ denotes the binary act $f$ such that $f(s)=x$ for $s \in A$, and $f(s)=y$ for $s \notin A$, where $A \in \Sigma$.

We model the DM's preferences on $\mathcal{F}$ by a binary relation $\succcurlyeq$. To rule out uninteresting cases, throughout the paper we require that $\succcurlyeq$ be nontrivial: There are $f, g \in \mathcal{F}$ such that $f \succ g$. Therefore, we henceforth call preference relation any non-trivial binary relation on $\mathcal{F}$. A functional $V: \mathcal{F} \rightarrow \mathbb{R}$ represents $\succcurlyeq$ if $V(f) \geq V(g)$ if and only if $f \succcurlyeq g$. Clearly, a necessary condition for $\succcurlyeq$ to have a representation is that it be a complete and transitive relation, so that, as customary, we can denote by $\sim$ and $\succ$ its symmetric and asymmetric components, respectively. A representation $V$ is monotonic if $V(f) \geq V(g)$ whenever $f, g \in \mathcal{F}$ are such that $f(s) \succcurlyeq g(s)$ for all $s \in S$.

Given a preference relation $\succcurlyeq$, acts $f, g \in \mathcal{F}$ are called comonotonic if there are no $s, s^{\prime} \in S$ such that $f(s) \succ f\left(s^{\prime}\right)$ and $g\left(s^{\prime}\right) \succ g(s)$. Finally, an event $A \in \Sigma$ is null (resp. universal) for a preference relation $\succcurlyeq$ if $y \sim x A y$ (resp. $x \sim x A y$ ) for all $x, y \in X$ such that $x \succ y$, while $A$ is essential for $\succcurlyeq$ if for some $x, y \in X$ we have $x \succ x A y \succ y$.

\subsection{Capacities and Choquet Integrals}

A set-function $\rho$ on $(S, \Sigma)$ is called a capacity if it is monotone and normalized, that is: if for $A, B \in \Sigma, A \subseteq B$, then $\rho(A) \leq \rho(B) ; \rho(\emptyset)=0$ and $\nu(S)=1$. A capacity is called a probability measure if it is (finitely) additive: For all $A, B \in \Sigma$ such that $A \cap B=\emptyset$, $\rho(A \cup B)=\rho(A)+\rho(B)$.

The notion of integral used for capacities is the Choquet integral, due to Choquet [8]. For a given function $\varphi \in B(\Sigma)$, the Choquet integral of $\varphi$ with respect to a capacity $\rho$ is defined as follows:

$$
\int_{S} \varphi d \rho=\int_{0}^{+\infty} \rho(\{s \in S: \varphi(s) \geq \alpha\}) d \alpha+\int_{-\infty}^{0}[\rho(\{s \in S: \varphi(s) \geq \alpha\})-1] d \alpha,
$$

where the integrals are taken in the sense of Riemann. When $\rho$ is additive, (2) is equal to a standard (additive) integral. In general, Choquet integrals are seen to be monotonic, positive homogeneous and comonotonic additive: If $\varphi, \psi \in B(\Sigma)$ are comonotonic, then $\int(\varphi+\psi) d \rho=\int \varphi d \rho+\int \psi d \rho$. 


\section{Biseparable Preferences}

The following definition is central to the paper. Given a representable preference relation, it singles out a subset of its representations. We call these representations canonical, since they are the ones which are looked at because of their separability properties:

Definition 1 Let $\succcurlyeq$ be a preference relation. We say that a representation $V: \mathcal{F} \rightarrow \mathbb{R}$ of $\succcurlyeq i s$ canonical if it is monotonic and there is a set-function $\rho_{V}: \Sigma \rightarrow[0,1]$ such that, if we let $u(x) \equiv V(x)$ for all $x \in X$, for all consequences $x \succcurlyeq y$ and all $A \in \Sigma$ we have:

$$
V(x A y)=u(x) \rho_{V}(A)+u(y)\left(1-\rho_{V}(A)\right) .
$$

The next result clarifies the roles of the functions $u$ and $\rho_{V}$.

Proposition 1 Let $\succcurlyeq$ be a preference relation with a canonical representation. For all its canonical representations $V$ and all $x, y \in X$,

$$
x \succcurlyeq y \Longleftrightarrow u(x) \geq u(y)
$$

Moreover, for all $x \succ y$ and all $A, B \in \Sigma$ we have

$$
x A y \succcurlyeq x B y \Longleftrightarrow \rho_{V}(A) \geq \rho_{V}(B) .
$$

That is, the index $u$ given by the restriction of $V$ to $X$ can be interpreted as the DM's (state-independent) utility function, so that we call it the canonical utility index of $\succcurlyeq$. The set-function $\rho_{V}$ is a numerical representation of the DM's 'likelihood' relation, that we call the DM's willingness to bet. ${ }^{8}$ It is the unique set-function that satisfies Eq. (3) for a given $V$. The next simple result presents some simple properties of the willingness to bet function $\rho_{V}$. In particular, it is worth noting that $\rho_{V}$ is a monotone set-function, i.e., a capacity.

Proposition 2 Let $V$ be a canonical representation of a preference relation $\succcurlyeq$. The set-function $\rho_{V}$ has the following properties:

(i) An event $A \in \Sigma$ is essential iff $\rho_{V}(A) \in(0,1)$.

(ii) An event $A \in \Sigma$ is not essential iff $\rho_{V}(A) \in\{0,1\}$. In particular, $\rho_{V}(A)=0$ iff $A$ is null, and $\rho_{V}(A)=1$ iff $A$ is universal.

(iii) If $A, B \in \Sigma$ are such that $A \subseteq B$, then $\rho_{V}(A) \leq \rho_{V}(B)$.

Remark 1 The proposition also shows that an event must be either null, universal, or essential. Moreover, it shows that for a preference relation with a canonical representation, $A$ is essential if and only if $x \succ x A y \succ y$ for every $x \succ y$.

\footnotetext{
${ }^{8}$ Notice that, in the presence of a canonical representation, this likelihood relation is independent of the stakes $x$ and $y$ (as long as $x \succ y$ ). See Axiom B4 below.
} 
In light of these results we can say that a DM whose preference $\succcurlyeq$ has a canonical representation chooses among binary acts as if he was maximizing the (Choquet) 'expectation' of the canonical utility $u$ with respect to the willingness to bet $\rho_{V}$. But his preferences over non-binary acts are not constrained to a specific functional form.

So far, we have not said anything about the uniqueness properties of canonical representations $V$, and hence of the canonical utilities $u$ and willingness to bet functions $\rho_{V}$. That is, given two canonical representations $V$ and $V^{\prime}$ of the same preference relation $\succcurlyeq$, are they related by more than just an increasing transformation? Clearly, if $V^{\prime}$ is a positive affine transformation of $V$ (i.e., there are $\alpha>0$ and $\beta \in \mathbb{R}$ such that $\left.V^{\prime}=\alpha V+\beta\right)$, it is a canonical representation. So canonical representations can at most be cardinal scales. In general, though, there is no reason to expect that they be cardinal. First of all, if $\succcurlyeq$ has no essential events, then Definition 1 does not impose any restriction on the structure of $V$ beyond monotonicity: Any increasing transformation of $V$ is also canonical (see also Proposition 6 below). Second, it is very simple to construct examples with finite $S$ and $X$ sets where $V$ and $V^{\prime}$ are canonical representations of $\succcurlyeq$ which are not related by an affine transformation (and they might even be expected utility functionals).

On the other hand, for interpretational purposes it is important that, when $\succcurlyeq$ has an essential event, its canonical representations be cardinal scales. First of all, because this implies that the utility index is a cardinal scale itself, so that for instance it makes sense to discuss its concavity (but see Theorem 2 below). Second, because this implies that the willingness to bet function is univocally determined, as we presently show in Proposition 3. Moreover, the class of preferences with a canonical representation which is not cardinal does not seem to contain many examples of interest. Indeed, we show later (Proposition 7) that such cardinality of the canonical representation (when it exists, and when there are essential events) is guaranteed for any preference satisfying a weak continuity condition.

We thus restrict our attention to the following subclass of preferences:

Definition 2 A preference relation $\succcurlyeq$ is called biseparable if it admits a canonical representation, and moreover such representation is unique up to positive affine transformations when $\succcurlyeq$ has at least one essential event.

As announced, biseparable preference relations have a unique willingness to bet:

Proposition 3 Let $\succcurlyeq$ be a biseparable preference relation. Then $\rho$ is unique: $\rho_{V}=\rho_{V^{\prime}}$ for all canonical representations $V$ and $V^{\prime}$ of $\succcurlyeq$.

Because of this result, we shall henceforth write $\rho$ instead of $\rho_{V}$ to denote the willingness to bet of a biseparable preference relation. We can thus conclude that biseparable preferences feature two important uniqueness properties: uniqueness up to positive affine transformations of $V$ (hence of $u$ ), and uniqueness of the willingness to bet function $\rho$. 
Remark 2 A converse question to the one about the uniqueness of $u$ and $\rho$ is the following: Often in applications it will be easier to identify these two functions than the overall preference functional $V$. Clearly, the same pair $(u, \rho)$ corresponds to a set of possible canonical representations. It is simple to see that this set can be formally described but, as the question is somewhat tangential to our focus here, we relegate this result to Appendix B.

Before showing that most of the decision models mentioned in the Introduction describe biseparable preferences, we look at a more special but popular decision setting, and present a variant of biseparability for that setting.

\subsection{Constant Linearity and the Anscombe-Aumann Setting}

An important special case of the decision setting we use is the one in which $X$ has a vector structure; precisely, it is a convex subset of a vector space. For instance, this is the case if $X$ is the set of all the lotteries on a set of prizes, as it happens in the classical setting of Anscombe and Aumann [2].

In this framework, it is natural and useful to consider the preferences satisfying the following condition: ${ }^{9}$

Definition 3 Let $X$ be a convex subset of a vector space. A canonical representation $V$ of a preference relation $\succcurlyeq$ is constant linear (c-linear, for short) if for all binary $f \in \mathcal{F}$, $x \in X$, and $\alpha \in[0,1]$,

$$
V(\alpha f+(1-\alpha) x)=\alpha V(f)+(1-\alpha) V(x) .
$$

A preference is called c-linear biseparable if it admits a c-linear canonical representation.

It is easy to verify that if a preference relation has a c-linear canonical representation, such representation is unique up to positive affine transformations. Therefore, for this class of preferences we do not have to specifically add a uniqueness requirement. It is important to observe that, their name notwithstanding, c-linear biseparable preference relations are not necessarily biseparable in the sense of Definition 2: They may have two canonical representations which are not related by a positive affine transformation (of course only one of them can be c-linear).

\section{$2.2 \quad$ Examples}

We claimed earlier that the class of biseparable preference relations contains a number of preference models with state-independent preferences. Here we partially substantiate

\footnotetext{
${ }^{9}$ As usual, for every $f, g \in \mathcal{F}$ and $\alpha \in[0,1], \alpha f+(1-\alpha) g$ is the act which pays $\alpha f(s)+(1-\alpha) g(s) \in X$ for every $s \in S$.
} 
this claim by showing that all these models induce a canonical representation. Later we show that when the consequence space $X$ is a connected and separable topological space and the $u$ function is continuous, they are all biseparable (Proposition 7 ). In the Anscombe-Aumann setting, they are all c-linear biseparable if the $u$ function is affine on $X$.

(i) Let $\succcurlyeq$ be a CEU ordering represented by the functional $V(f)=\int u(f) d \nu$, where $\nu$ is a capacity, and the integral is taken in the sense of Choquet. It is immediate to check that $V$ is a canonical representation of $\succcurlyeq$, and $u$ is a canonical utility index of $\succcurlyeq$. In fact, monotonicity is well known, and Eq. (3) holds with $\rho(A)=\nu(A)$ for all $A \in \Sigma$.

A special type of CEU preferences are the rank-dependent EU (RDEU) preferences, for which the capacity $\rho$ is a 'distortion' $g(P)$ of some probability $P$. SEU orderings correspond to the special case of RDEU in which $g$ is the identity. Clearly, both have a canonical representation.

(ii) A popular generalization of CEU is the cumulative prospect theory (CPT) of Tversky and Kahneman [29]. In CPT some consequence is established to be the DM's reference point. The consequences which are better than the reference point are called gains, and those which are worse are called losses. The preferences over $\mathcal{F}$ are represented as follows: Given a utility function $u$, normalized so that the reference point has utility 0 , every act $f$ is split into its 'gain' part $f^{+}$(of the payoffs with positive utility) and its 'loss' part $f^{-}$(of the payoffs with negative utility). $V(f)$ is the sum of the Choquet integral of $u\left(f^{+}\right)$w.r.t. a capacity $\nu^{+}$and the Choquet integral of $u\left(f^{-}\right)$w.r.t. another capacity $\nu^{-}$. (CEU corresponds to the special case in which $\nu^{-}=\nu^{+}$.) A CPT preference relation has a canonical representation only if it is also CEU. However, a CPT preference has a canonical representation on the sets of acts which only yield gains (or only losses).

(iii) Let $\succcurlyeq$ be a preference relation represented by a MEU preference functional $V(f)=$ $\min _{P \in C} \int u(f) d P$, with $C$ a closed and convex set of probability measures. It is easy to check that $V$ is a canonical representation of $\succcurlyeq$, and $u$ is a canonical utility index of $\succcurlyeq$. In fact, monotonicity is immediate, and Eq. (3) is seen to hold with $\rho(A)=\min _{P \in C} P(A)$ for all $A \in \Sigma$.

More generally, consider a preference $\succcurlyeq$ which may not be as 'conservative' as MEU predicts. That is, there is $\alpha \in[0,1]$, such that $\succcurlyeq$ is represented by the following preference functional, ${ }^{10}$

$$
V(f)=\left[\alpha \min _{P \in C} \int_{S} u(f(s)) P(d s)+(1-\alpha) \max _{P \in C} \int_{S} u(f(s)) P(d s)\right] .
$$

$V$ is a canonical representation of $\succcurlyeq$ : Eq. (3) holds with $\rho$ defined for every $A \in \Sigma$ by

$$
\rho(A)=\alpha \min _{P \in C} P(A)+(1-\alpha) \max _{P \in C} P(A) .
$$

10 This includes the case of a 'maximax' DM, who has $\alpha=0$. 
(iv) Consider a DM who behaves as if he was maximizing SEU when choosing among binary acts, but not when comparing more complex acts. Formally, there is a utility $u$, a probability $P$ and a number $\beta \in[0,1]$ such that the DM's preference relation $\succcurlyeq$ is represented by

$$
V(f) \equiv(1-\beta) \int_{S} u(f(s)) P(d s)+\beta \varphi_{*}(u(f)),
$$

where for every $\phi \in B(\Sigma)$,

$$
\varphi_{*}(\phi)=\sup \left\{\int \phi^{\prime} d P: \phi^{\prime} \in B(\Sigma) \text { is two-valued, } \phi^{\prime}(s) \leq \phi(s) \text { for all } s \in S\right\} \text {. }
$$

$V$ is a canonical representation of $\succcurlyeq$ : Monotonicity is straightforward, and Eq. (3) holds with $\rho=P$ (i.e., $P$ represents $\succcurlyeq$ 's willingness to bet).

More generally, let $N: B(\Sigma) \rightarrow \mathbb{R}$ be a functional which is non-negative, monotonic, and continuous when $B(\Sigma)$ is endowed with the sup-norm topology. Let $\beta: B(\Sigma) \rightarrow$ $\mathbb{R}$ be defined as follows: $\beta(\phi)=1 /(1+N(\phi))$. Consider a preference $\succcurlyeq$ which is represented as above, where $\beta(u(f))$ replaces the constant $\beta$. It can be seen that $V$ is a canonical representation of $\succcurlyeq$ (see Example 1 for details).

\subsection{First Behavioral Properties}

Preferences with a canonical representation satisfy some simple behavioral properties that for convenience we now list as axioms. First of all, biseparable preferences are weak orders, i.e., complete and transitive relations.

B1 (Weak order) (a) For all $f, g \in \mathcal{F}, f \succcurlyeq g$ or $g \succcurlyeq f$. (b) For all $f, g, h \in \mathcal{F}$, if $f \succcurlyeq g$ and $g \succcurlyeq h$, then $f \succcurlyeq h$.

Biseparable preferences also satisfy the next two mild monotonicity axioms. Both axioms are widely used in the literature, and imply a form of state independence. Notice that B3, which is a very weak version of Savage's P3 [27], is a converse to B2 for some binary acts.

B2 (Statewise Dominance) For every $f, g \in \mathcal{F}$, if $f(s) \succcurlyeq g(s)$ for every $s \in S$, then $f \succcurlyeq g$.

B3 (Eventwise Monotonicity) For every non-null $A \in \Sigma$ and every $x, y \succcurlyeq z \in X$,

$$
x \succ y \Longleftrightarrow x A z \succ y A z .
$$

For every non-universal $A \in \Sigma$ and every $x, y \preccurlyeq z \in X$,

$$
x \succ y \Longleftrightarrow z A x \succ z A y .
$$


Recalling Remark 1, axiom B1 is immediately seen to be responsible for the fact that an event is either one of null, essential or universal. In contrast, axiom B3 can be used to show directly that an event $A$ is essential if and only if $x \succ x A y \succ y$ for all $x \succ y$.

As to 'betting' behavior, biseparable preferences also satisfy the following standard comparative probability axiom. This is not used in the axiomatizations later, since it is implied by the other axioms, but it is a well understood property (it is Savage 's P4 axiom [27]).

B4 (Comparative Probability) For every $A, B \in \Sigma$ and all $x, x^{\prime}, y, y^{\prime} \in X$ such that $x \succ y$ and $x^{\prime} \succ y^{\prime}$,

$$
x A y \succcurlyeq x B y \Longleftrightarrow x^{\prime} A y^{\prime} \succcurlyeq x^{\prime} B y^{\prime}
$$

Axiom B4 owes its name to the fact that it implies that the DM's preferences over 'bets' are independent of the relative size of the stakes, so that we can define the DM's 'likelihood' (comparative probability) ordering by looking at his willingness to bet.

Remark 3 In the presence of axiom B4, it is equivalent to define an event $A$ null (resp. universal) if $y \sim x A y$ for some $x \succ y$. This does make axiom B3 weaker (in isolation). However, in the context of our axiomatizations, requiring B1-B4 with this weak notion of null/universal is equivalent to requiring B1-B3 with the strong notion that we use.

\section{Risk Aversion and the Canonical Utility Function}

In this section, we show that some well-known characterizations of risk aversion - that have been proved for SEU or some other preference models that belong to the biseparable class - can be extended to biseparable preference relations. ${ }^{11}$ We present three different approaches. We start with a general comparative approach, in an arbitrary consequence space. Then, we restrict our attention to the important case of monetary consequences, and analyse risk attitude by looking at preference for diversification and mean-preserving spreads.

In reading these results, it is important to keep in mind that we provide here different characterizations of what in the Introduction we called 'cardinal' risk aversion. The latter is narrower than the 'risk aversion' notion used in the literature on non-SEU preferences, even though the two notions coincide for SEU preferences. In the SEU case, many manifestations of risk aversion are equivalent to the concavity of the von Neumann-Morgenstern utility index. We show that analogous results are true for biseparable preferences. Interestingly, these results are generalizations of the corresponding results for SEU preferences, showing that concavity follows from weaker conditions than commonly assumed.

\footnotetext{
11 Needless to say, symmetric results could be proved about cardinal risk loving.
} 


\subsection{The Comparative Approach}

In the comparative approach, we depart from a comparison of the relative cardinal risk aversion of two DMs. Consider the following notion, that we introduced in [15]:

Definition 4 Let $\succcurlyeq_{1}$ and $\succcurlyeq_{2}$ be two weak orders. We say that $\succcurlyeq_{2}$ is more uncertainty averse than $\succcurlyeq_{1}$ if: For all $x \in X$ and $f \in \mathcal{F}$, both

$$
x \succcurlyeq_{1} f \Longrightarrow x \succcurlyeq_{2} f
$$

and

$$
x \succ_{1} f \Longrightarrow x \succ_{2} f
$$

Though compelling and natural, this comparative notion does not distinguish between ambiguity and cardinal risk attitudes. In [15] we proposed a way to coarsen this ranking so as to make sure that we are only comparing the DMs' ambiguity attitude. In contrast, here we need to coarsen the relative uncertainty aversion ranking so as to avoid that differences in the DMs' ambiguity attitude (in the wide sense discussed in footnote 4) intrude in the comparison. A natural way to do this is suggested by the traditional treatment of risk aversion in the EU setting: Compare DMs who have identical beliefs, i.e., a common willingness to bet $\rho$ (cf. Yaari [31, Remark 1]).

Definition 5 Let $\succcurlyeq_{1}$ and $\succcurlyeq_{2}$ be two biseparable preference relations. We say that $\succcurlyeq_{2}$ is more cardinal risk averse than $\succcurlyeq_{1}$ whenever both the following conditions hold:

1. $\succcurlyeq_{2}$ is more uncertainty averse than $\succcurlyeq_{1}$;

2. $\succcurlyeq_{1}$ and $\succcurlyeq_{2}$ have a common willingness to bet $\rho$.

Given this relative notion, it is now theoretically straightforward to provide an absolute notion of cardinal risk aversion: Establish arbitrarily that certain benchmark preferences are to be classified as 'cardinal risk neutral', and then say that a preference is cardinal risk averse if it is more cardinal risk averse than some cardinal risk neutral preference. Practically, though, there is no 'obvious' choice of benchmark preferences until we say something more about $X$ (as we shall do in the next subsection).

However, the next result shows that the relative notion can be characterized immediately: 'More cardinal risk averse' in this sense implies 'more concave', as in the standard SEU case. Here we say that two utility indices are commonly normalized on a pair of points in $X$, if they are set to be equal to the same values ( 0 and 1$)$ on those two points.

Theorem 1 Suppose that $X$ is a connected topological space, and let $\succcurlyeq_{1}$ and $\succcurlyeq_{2}$ be two biseparable preference relations, with continuous canonical utility indexes $u_{1}$ and $u_{2}$ respectively. ${ }^{12}$ Consider the following statements:

\footnotetext{
${ }^{12}$ For example, this is the case if $X$ is also separable and $\succcurlyeq_{1}$ and $\succcurlyeq_{2}$ satisfy axiom S1 below, or if they are c-linear biseparable in an Anscombe-Aumann setting.
} 
(i) $\succcurlyeq_{2}$ is more cardinal risk averse than $\succcurlyeq_{1}$;

(ii) $\succcurlyeq_{1}$ and $\succcurlyeq_{2}$ are ordinally equivalent, and for any pair $x \succ y$, if we commonly normalize on $x$ and $y$, we have for every $z \in X$ such that $x \succcurlyeq z \succcurlyeq y, u_{2}(z) \geq u_{1}(z)$;

(iii) $u_{2}$ is an increasing concave transformation of $u_{1}$.

They are related as follows: $(i) \Rightarrow($ ii $) \Leftrightarrow($ iii $)$.

Remark 4 Notice that differently from the standard result for SEU preferences, we do not assume any order or metric structure on $X$, or differentiability of the utility functions. It is well-known (de Finetti [9], Arrow [3], Pratt [24]) that in the case in which $X \subseteq \mathbb{R}$ and the utility functions have differentiability properties, (iii) is also equivalent to pointwise dominance of the Arrow-Pratt coefficients of absolute risk aversion. Yaari [31] uses differentiability assumptions to prove a result different from ours, but in the same spirit: For a class of preferences which roughly contains the biseparable ones, he shows that the relative cardinal risk aversion ranking is characterized by a natural measure of local risk aversion (which in the SEU case is equivalent to the Arrow-Pratt measure).

We now look at the converse of Theorem 1, and ask when 'more concavity' means 'more cardinal risk aversion'. Answering this question requires adding structure to the DMs' preferences over non-binary acts, as described by the following definition. First, we state a simple and general result:

Lemma 1 Let $\succcurlyeq$ be a representable preference relation. Then $V: \mathcal{F} \rightarrow \mathbb{R}$ is a monotonic representation of $\succcurlyeq$ if and only if there exists a monotone functional $I_{V}: B(\Sigma) \rightarrow \mathbb{R}$ such that $V(f)=I_{V}(u(f))$, where $u(x) \equiv V(x)$ for all $x \in X$.

In particular, the lemma implies that for any canonical representation $V$ of a biseparable preference relation, we can find a functional $I_{V}$ which 'generates' $V$ from $u$. For instance, if $\succcurlyeq$ is a CEU preference with utility $u$ and capacity $\nu$, for the canonical representation $V(f)=\int u(f) d(\nu)$ we have $I_{V}(\phi)=\int \phi d \nu$. In light of this, we can now state a key property that relates two biseparable preferences.

Definition 6 Two biseparable preferences $\succcurlyeq_{1}$ and $\succcurlyeq_{2}$ have a common canonical functional if we can find two canonical representations $V_{1}$ and $V_{2}$, of $\succcurlyeq_{1}$ and $\succcurlyeq_{2}$ respectively, such that $u_{1}(X) \cap u_{2}(X) \neq \emptyset$ and $V_{1}=I^{*}\left(u_{1}\right)$ and $V_{2}=I^{*}\left(u_{2}\right)$ for the same monotone functional $I^{*}: B(\Sigma) \rightarrow \mathbb{R}$.

Two biseparable preferences that have a common canonical functional share the same willingness to bet, and also belong to the same 'class'; e.g., they are both SEU, CEU, or MEU. Conversely, if $\succcurlyeq_{1}$ and $\succcurlyeq_{2}$ are either both CEU (or both MEU), then they have a common canonical functional, and they can be shown to satisfy the condition on the ranges as well. 
Proposition 4 Under the hypotheses of Theorem 1, (iii) implies (i) if the two preference relations $\succcurlyeq_{1}$ and $\succcurlyeq_{2}$ have a common canonical functional $I^{*}: B(\Sigma) \rightarrow \mathbb{R}$ that satisfies the following (Jensen) property

$$
\psi\left(I^{*}(\phi)\right) \geq I^{*}(\psi(\phi))
$$

for all $\phi \in B(\Sigma)$ and all increasing concave $\psi \in B(\Sigma)$.

The Jensen property of Eq. (7) is satisfied by a number of preference functionals of interest; for instance, the CEU integrals and the MEU representation functionals. We therefore have that if two preferences are both CEU (or both MEU) and the assumptions of Theorem 1 are satisfied, all the statements in that theorem are equivalent.

\subsection{Monetary Payoffs}

Turn now to the case in which consequences are monetary; i.e., $X$ is an interval of $\mathbb{R}$. In this case, there is an 'obvious' way to obtain the absolute notion of cardinal risk aversion discussed in the previous subsection: Define 'cardinal risk neutral' any preference for which $u$ is affine (so that on binary acts, it maximizes Choquet expected value). It then follows from Theorem 1 that a biseparable preference is cardinal risk averse only if its canonical utility index is increasing and concave (which becomes an 'if' under the assumptions of Proposition 4). Indeed, in this case we can also obtain some other interesting characterizations of cardinal risk aversion, that we describe in the rest of this subsection.

\subsubsection{Preference for Diversification}

We start with the extension of an interesting result of Chateauneuf and Tallon [6, Theorem 3.3] to preference relations with a canonical representation (they prove it for CEU preferences). The following is a weak version of a property studied by Dekel [11], which enjoys the conceptual advantage over the other notions presented in this section of being defined only in terms of primitives. It is implied by the notion of preference for comonotone diversification that is used in [6].

Definition 7 A preference relation exhibits preference for bet diversification if, for every essential event $A \in \Sigma$ and $x, x^{\prime}, y, y^{\prime} \in X$, with $x \succ y$ and $x^{\prime} \succ y^{\prime}$, we have for every $\alpha \in[0,1]$,

$$
x A y \sim x^{\prime} A y^{\prime} \Longrightarrow\left[\alpha x+(1-\alpha) x^{\prime}\right] A\left[\alpha y+(1-\alpha) y^{\prime}\right] \succcurlyeq x A y .
$$

A DM exhibiting preference for bet diversification prefers bets with a smoother payoff profile, when betting on the same essential event. Intuitively, this is a feature that we would relate to the DM's risk aversion. It is seen to correspond to the concavity of $u$ : 
Theorem 2 Suppose that $X$ is an interval of $\mathbb{R}$, and let $\succcurlyeq$ be a preference relation with a canonical representation whose canonical utility function $u$ is continuous. Then, $\succcurlyeq$ exhibits preference for bet diversification if and only if $u$ is concave.

\subsection{2 'Subjective' Expected Values and Mean Preserving Spreads}

The two most popular definitions of risk aversion in the literature hinge crucially on the existence of an external notion of 'objective' probability. The first one is the traditional 'internal' definition, that says that a preference is risk averse if it prefers the expected value of a lottery to the lottery itself. The second definition, which can also be applied to non-EU preferences, is that which defines risk averse a preference which between any pairs of lotteries, such that one of the two is a mean-preserving spread of the other, prefers the less disperse one. Clearly, in general the latter is more demanding than the former. In the EU case, they are both characterized by concavity of $u$.

In the Anscombe-Aumann framework, where we interpret the convexity of $X$ as arising from the existence of an external randomizing device, similar results hold for c-linear biseparable preferences, with identical definitions and characterization. Formulating such notions in a purely subjective setting is not as straightforward, since it is not clear how to obtain expected values and mean-preserving spreads. However, when dealing with biseparable preferences, we can use the DM's subjective beliefs to obtain 'subjective' expected values and mean-preserving spreads for binary acts. (Clearly, we do not have any basis for calculating the subjective expected value or mean-preserving spreads of nonbinary acts.)

We thus have the following version of the first definition of risk aversion, where for any biseparable preference with willingness to bet $\rho$ and act $f=x A y$ with $x \succcurlyeq y$, we let $E V(f) \equiv x \rho(A)+y(1-\rho(A)) \in X$, the subjective expected value of $f$.

Definition 8 Given a biseparable preference $\succcurlyeq$ with willingness to bet $\rho$, we say that $\succcurlyeq$ has a preference for the subjective expected value if for every binary act $f=x A y$, we have that

$$
E V(f) \succcurlyeq f
$$

To introduce a version of the mean-preserving definition of risk aversion, we first need to define a (binary) subjective mean-preserving spread of a given binary act.

Definition 9 Given a biseparable preference relation $\succcurlyeq$ with willingness to bet $\rho$, and a bet $x A y$ with $x \succcurlyeq y$, we say that $f \in \mathcal{F}$ is a binary subjective mean-preserving spread (SMPS) of $x A y$ (for $\succcurlyeq$ ) if there are $x^{\prime}, y^{\prime} \in \mathcal{X}$ with $x^{\prime} \succcurlyeq x$ and $y \succcurlyeq y^{\prime}$, and $B \in \Sigma$ with $\rho(A)=\rho(B)$, such that $f=x^{\prime} A y^{\prime}$ and

$$
x^{\prime} \rho(B)+y^{\prime}(1-\rho(B))=x \rho(A)+y(1-\rho(A)) .
$$


Clearly, both the subjective notions of expected value and MPS coincide with their traditional counterparts when there is an external probability, and $\rho$ coincides with it. We can now reinforce Definition 8 as follows:

Definition 10 A biseparable preference relation $\succcurlyeq i s$ averse to binary SMPSs if it weakly prefers $x A y$ (with $x \succcurlyeq y$ ) to every one of its binary SMPSs.

The following straightforward result shows that under a weak assumption both these behavioral features are equivalent to the concavity of the canonical utility index:

Proposition 5 Suppose that $X$ is an interval of $\mathbb{R}$, and let $\succcurlyeq$ be a biseparable preference relation. Consider the following statements:

(i) The canonical utility index $u$ is concave;

(ii) $\succcurlyeq$ is averse to binary SMPSs;

$($ iii $) \succcurlyeq$ has a preference for the subjective expected value.

We have $(i) \Rightarrow($ ii $) \Rightarrow($ iii $)$, while $($ iii $) \Rightarrow(i)$ holds whenever $\rho(\Sigma)=[0,1]$.

\section{Axiomatization}

We now turn to the critical issue of the axiomatic characterization of biseparable preferences. We begin with the characterization of c-linear biseparable preferences in the 'Anscombe-Aumann' setting in which $X$ is a convex subset of a vector space, and then provide a characterization of biseparable preferences in the more general Savage setting.

\subsection{The Anscombe-Aumann Case}

Consider the following axioms, some of which exploit the vector structure of $X$ :

A1 (Certainty Equivalents) For all $f \in \mathcal{F}$, there is $x \in X$ such that $x \sim f$.

A2 (Weak Certainty Independence) For all binary $f, g \in \mathcal{F}$ and all $x \in X$, if $\alpha \in$ $(0,1)$ then

$$
f \succ g \Longleftrightarrow \alpha f+(1-\alpha) x \succ \alpha g+(1-\alpha) x
$$

A3 (Archimedean Axiom) For all $x, y, z \in X$, if $x \succ y \succ z$ then there exist $\alpha, \beta \in$ $(0,1)$ such that

$$
\alpha x+(1-\alpha) z \succ y \succ \beta y+(1-\beta) z .
$$


All these axioms are mild behavioral assumptions. Axioms A1 and A3 are standard and play mostly a technical role. Axiom A2 is a very weak and compelling version of the independence axiom: It only requires that independence holds whenever we are comparing binary acts, and we are 'mixing' them with a constant act. It is a weakening of the 'certainty independence' axiom introduced by Gilboa and Schmeidler [18] as the cornerstone of their axiomatization of MEU in the Anscombe-Aumann framework. As it turns out, alongside B1-B3, these three axioms characterize c-linear biseparable preference relations.

Theorem 3 Suppose that $X$ is a convex subset of a vector space, and let $\succcurlyeq$ be a preference relation on $\mathcal{F}$. Then the following statements are equivalent:

$(i) \succcurlyeq$ satisfies axioms $B 1-B 3$ and $A 1-A 3$.

(ii) There exist a monotonic representation $V: \mathcal{F} \rightarrow \mathbb{R}$ of $\succcurlyeq$ and a capacity $\rho: \Sigma \rightarrow$ $[0,1]$ such that:

- for all $x \succcurlyeq y$ in $X$, all $A \in \Sigma$, letting $u(x) \equiv V(x)$ for all $x \in X$, we have

$$
V(x A y)=u(x) \rho(A)+u(y)(1-\rho(A)) ;
$$

- for all binary $f \in \mathcal{F}$ and $x \in X$, and all $\alpha \in[0,1]$,

$$
V(\alpha f+(1-\alpha) x)=\alpha V(f)+(1-\alpha) V(x) .
$$

Moreover, the representation $V$ is unique up to positive affine transformations and the capacity $\rho: \Sigma \rightarrow[0,1]$ is unique.

\subsection{The Savage Case}

The characterization of biseparable preference relations is less straightforward in the more general Savage setting. Here it is useful to distinguish between the cases of preferences with and without essential events.

\subsubsection{Without Essential Events}

Starting with the latter case, we have a very general result: Under axioms B1-B3, if $\succcurlyeq$ has no essential events, every representation is canonical. Therefore, every preference relation satisfying these axioms is biseparable provided it admits a representation (which is the case, e.g., if it satisfies axiom S1 below).

Proposition 6 Let $\succcurlyeq$ be a preference relation satisfying axioms B1-B3. If $\succcurlyeq$ has no essential events, every functional $V: \mathcal{F} \rightarrow \mathbb{R}$ representing $\succcurlyeq$ has the following (Choquet) integral representation: for every $f \in \mathcal{F}$,

$$
V(f)=\int_{S} u(f(s)) \rho(d s),
$$


where $\rho$ is the dichotomous capacity on $\Sigma$ defined by $\rho(A)=0$ if $A$ is null, $\rho(A)=1$ if $A$ is universal, and $u: X \rightarrow \mathbb{R}$ is defined by $u(x) \equiv V(x)$ for every $x \in X$.

Observe that Eq. (9) reduces to (3) for binary acts $x A y$, with $x \succcurlyeq y$. Hence, every $V$ representing $\succcurlyeq$ is canonical: With no essential events the canonical representations of preferences satisfying B2 and B3 are only unique up to normalizable increasing transformations.

Quite surprisingly, Eq. (9) shows that under the assumptions of the proposition, the preference relation has a Choquet integral representation for all, rather than just the binary, acts. Hence, any representable preference relation $\succcurlyeq$ which satisfies statewise dominance, eventwise monotonicity and does not have essential events has a CEU representation.

\subsubsection{With Essential Events}

In the case in which $\succcurlyeq$ has some essential events, we begin by endowing $X$ with a topology $\tau$. In turn, $\tau$ induces the product topology on the set $X^{S}$ of all functions from $S$ into $X$. In this topology, a net $\left\{f_{\alpha}\right\}_{\alpha \in D} \subseteq X^{S}$ converges to $f \in X^{S}$ if and only if $f_{\alpha}(s) \stackrel{\tau}{\longrightarrow} f(s)$ for all $s \in S$ (remember that $S$ is arbitrary). For this reason it also called the topology of pointwise convergence (nets are needed since the topology might not be first countable). We now have:

S1 (Continuity) Let $\left\{f_{\alpha}\right\}_{\alpha \in D} \subseteq \mathcal{F}$ be a net that pointwise converges to $f \in \mathcal{F}$, and such that all $f_{\alpha}$ 's and $f$ are measurable with respect to the same finite partition. If $f_{\alpha} \succcurlyeq g$ (resp. $g \succcurlyeq f_{\alpha}$ ) for all $\alpha \in D$, then $f \succcurlyeq g$ (resp. $\left.g \succcurlyeq f\right)$.

$\mathrm{S} 1$ is a standard continuity axiom. It is very weak, as the clause that all acts in the net used in the axiom are measurable w.r.t. the same partition significantly limits its demands. In particular, it is substantially weaker than requiring continuity w.r.t. to the product topology.

It is easy to see (Lemma 4 in Appendix C.1) that any preference relation satisfying B1, B2 and S1 on a connected $X$ has certainty equivalents. That is, it satisfies axiom A1 above. Granted this, we henceforth denote by $c_{f}$ an arbitrarily chosen certainty equivalent of $f \in \mathcal{F}$.

Another consequence of S1 is the following result, that was anticipated in Section 2.

Proposition 7 Suppose that $X$ is a connected and separable space, and let $\succcurlyeq$ be a preference relation with a canonical representation. Then if $\succcurlyeq$ satisfies $S 1$, it is biseparable.

That is, when continuity holds, canonical representations are unique up to normalizable positive affine transformations (in the presence of an essential event). This result can be 
used to show that all the preferences in examples (i)-(iv) of subsection 2.2 are biseparable: Just assume that $X$ is a connected and separable topological space, and that $u$ is continuous in this topology.

The characterization of biseparable preferences requires two more axioms with more substantial empirical content. The first one is a separability property. We write $\{x, y\} \succcurlyeq$ $z$ as a short-hand for $x \succcurlyeq z$ and $y \succcurlyeq z$ (and similarly later for $\succ$ and $\prec$ ).

S2 (Binary Comonotonic Act Independence) For every essential $A \in \Sigma$, every $B \in\left\{A, A^{c}\right\}$, and for all $f, g, h \in \mathcal{F}$ such that $f=x A y, g=x^{\prime} A y^{\prime}$ and $h=x^{\prime \prime} A y^{\prime \prime}$. If $f, g, h$ are pairwise comonotonic, and $\left\{x, x^{\prime}\right\} \succcurlyeq x^{\prime \prime}$ and $\left\{y, y^{\prime}\right\} \succcurlyeq y^{\prime \prime}$ (or $x^{\prime \prime} \succcurlyeq\left\{x, x^{\prime}\right\}$ and $\left.y^{\prime \prime} \succcurlyeq\left\{y, y^{\prime}\right\}\right)$, then

$$
x A y \succcurlyeq x^{\prime} A y^{\prime} \Longrightarrow\left[c_{\left(x B x^{\prime \prime}\right)} A c_{\left(y B y^{\prime \prime}\right)}\right] \succcurlyeq\left[c_{\left(x^{\prime} B x^{\prime \prime}\right)} A c_{\left(y^{\prime} B y^{\prime \prime}\right)}\right]
$$

$\mathrm{S} 2$ is a weak subjective version of the well known independence axiom of EU, and of Schmeidler's 'comonotonic independence' [28] axiom: ${ }^{13}$ First of all, notice that it only applies to binary acts measurable with respect to the algebra $\Sigma_{A} \equiv\left\{\emptyset, A, A^{c}, S\right\}$, with $A$ an essential event. To have a better intuition of what the axiom says, notice that even if we do not have access to a randomizing device, we can still construct a subjective 'mixture' of two acts $f$ and $g$ as follows: Fix some essential event $A$, and then construct state by state the act which for every state $s$ yields the certainty equivalent of the bet $f(s) A g(s)$. If $g$ is statewise dominated by $f$, the constructed act's payoffs are all indifferent to bets on the event $A$, so that it is analogous to a mixture in the AnscombeAumann framework. In the spirit of comonotonic independence, S2 requires that 'mixing' in this fashion with a comonotonic act $h$ does not affect the ranking of the two acts $f$ and $g$. The additional requirement that $h$ be either state by state better, or worse, than the other two acts guarantees that the 'mixing' operation is performed correctly, i.e., without involving bets on different events $\left(B\right.$ and $\left.B^{c}\right)$.

The last axiom guarantees that when evaluating bets, the DM's cardinal risk attitude (as defined in the Introduction) is independent of the event he is betting on.

S3 (Cardinal State Independence) For every essential $A \in \Sigma$ and for all $x, y, x^{\prime}, y^{\prime} \in$ $X$, if there are $m_{1}^{\prime} \prec m_{1}^{\prime \prime} \prec\left\{x, x^{\prime}\right\}$ and $m_{2}^{\prime} \prec m_{2}^{\prime \prime} \prec\left\{x, x^{\prime}\right\}$ and essential $B \in \Sigma$ such that

$$
x A m_{1}^{\prime \prime} \sim y A m_{1}^{\prime} \quad \text { and } \quad x B m_{2}^{\prime \prime} \sim y B m_{2}^{\prime},
$$

then

$$
x^{\prime} A m_{1}^{\prime \prime} \sim y^{\prime} A m_{1}^{\prime} \quad \text { if and only if } \quad x^{\prime} B m_{2}^{\prime \prime} \sim y^{\prime} B m_{2}^{\prime} .
$$

If, instead, there are $m_{1}^{\prime} \succ m_{1}^{\prime \prime} \succ\left\{x, x^{\prime}\right\}$ and $m_{2}^{\prime} \succ m_{2}^{\prime \prime} \succ\left\{x, x^{\prime}\right\}$ and essential $B$ such that (10) holds with $\left(A^{c}, B^{c}\right)$ in place of $(A, B)$, then (11) holds with the same substitution.

\footnotetext{
${ }^{13}$ Indeed, it is a weakening of the 'comonotonic act-independence' axiom of Chew and Karni [7], based on earlier work of Gul [19] and Nakamura [22].
} 
Like axiom S2, axiom S3 only applies to binary acts. ${ }^{14}$ In the presence of axiom B3, all these acts are comonotonic, since they are bets 'on' the events $A$ or $B$ : the payoff to $A$ (or $B$ ) obtaining is higher than that to $A^{c}$ (or $B^{c}$ ) obtaining.

To understand the role of this axiom, assume for concreteness that consequences are monetary payoffs, and that the DM likes more money to less. Suppose that, when betting on events $A$ and $B$ respectively, (10) holds for some 'win' payoffs $x$ and $y$ and 'loss' payoffs $m_{1}^{\prime \prime} \succ m_{1}^{\prime}$ and $m_{2}^{\prime \prime} \succ m_{2}^{\prime}$ respectively. This says that a reduction in the 'loss' payoffs from $m_{1}^{\prime \prime}$ to $m_{1}^{\prime}$, and from $m_{2}^{\prime \prime}$ to $m_{2}^{\prime}$, can be traded off in both cases with a reduction in 'win' from $x$ to $y .{ }^{15}$ Suppose that when the initial win is $x^{\prime}<x$, for bets on $A$ the DM is willing to trade off the decrease of the 'loss' with an increase of the 'win' to $y$ ', but for bets on $B$, the DM requires increasing the 'win' to $y^{\prime \prime}>y^{\prime}$ (that is, $x^{\prime} B m_{2}^{\prime \prime} \succ y^{\prime} B m_{2}^{\prime}$, in violation of (11)). That is, the DM is more sensible to differences in payoffs when betting on $B$ than when betting on $A$. Such 'inconsistency' of the DM's cardinal risk attitude is ruled out by cardinal state independence, which requires that the DM consistently evaluates the acceptable tradeoff for improving his 'win' payoff, and similarly for the 'loss' payoff.

We can now state the result for the essential case. In this result, we say that the preference functional $V: \mathcal{F} \rightarrow \mathbb{R}$ is sub-continuous if $\lim _{\alpha} V\left(f_{\alpha}\right)=V(f)$ whenever $\left\{f_{\alpha}\right\}_{\alpha \in D} \subseteq \mathcal{F}$ is a net that pointwise converges to $f \in \mathcal{F}$, and such that all $f_{\alpha}$ 's and $f$ are measurable with respect to the same finite partition. Notice that this implies that $u$ is $\tau$-continuous.

Theorem 4 Let $\succcurlyeq$ be a preference relation with some essential event. If $X$ is a connected and separable topological space, the following conditions are equivalent:

$(i) \succcurlyeq$ satisfies B1-B3 and S1-S3.

(ii) There exist a sub-continuous monotonic representation $V: \mathcal{F} \rightarrow \mathbb{R}$ of $\succcurlyeq$ and a capacity $\rho: \Sigma \rightarrow[0,1]$ such that: for all $f \in \mathcal{F}$, all $x \succcurlyeq y$ in $X$, all $A \in \Sigma$, letting $u(x) \equiv V(x)$ for all $x \in X$,

$$
V(x A y)=u(x) \rho(A)+u(y)(1-\rho(A)) .
$$

Moreover, the representation $V$ is unique up to positive affine transformations and the capacity $\rho: \Sigma \rightarrow[0,1]$ is unique.

Summing up, axioms B1-B3 and S1-S3 are sufficient for showing that a preference relation is biseparable when the space of consequences has some topological structure (whether or not there is an essential event). In general, axioms B1-B3 and S2-S3 are necessary; axiom S1 is also necessary if one of the canonical representations is subcontinuous.

${ }^{14}$ Axiom S3 is inspired by the techniques of additive conjoint measurement (see Krantz et al. [20] and Wakker [30]).

${ }^{15}$ We stress that the following discussion makes sense only when the DM is faced with nontrivial uncertainty (i.e., he is betting on essential events). Thus, we use 'trade-off' not to mean certain substitution, but substitution in the context of an uncertain prospect. 


\subsection{Probabilistic Beliefs}

We conclude our characterization section with a brief observation on how to axiomatically derive probabilistic beliefs. That is, we want to find conditions that deliver a biseparable preference whose representing capacity $\rho$ is a probability measure. It would be straighforward to obtain finite additivity of $\rho$ by adding a preference version of the classical necessary and sufficient condition for representability of a qualitative probability of, e.g., Chateauneuf [5]. For the sake of brevity, here we take a different route which we find more estethically appealing: We derive axiomatically a weak additivity property, and we also assume that the preference in question is either ambiguity averse or loving, according to the behavioral notion that we proposed in [15].

Recall from the Introduction that if a biseparable preference $\succcurlyeq$ with willingness to bet $\rho$ is ambiguity averse (resp. loving) in that sense, then [15, Theorem 3] there is a probability measure $P$ on $\Sigma$ such that $P(A) \geq \rho(A)$ (resp. such that $\rho(A) \geq P(A)$ ) for all $A \in \Sigma$. Now, say that a capacity $\rho$ is complement additive if for all $A \in \Sigma, \rho(A)+\rho\left(A^{c}\right)=$ 1. It is easy to show that a complement symmetric capacity that setwise dominates (or is dominated by) a probability must be identical to that probability. Thus, if we can show that a biseparable preference $\succcurlyeq$ induces a complement symmetric willingness to bet, then adding the assumption that $\succcurlyeq$ is ambiguity averse or loving is sufficient to show that it has probabilistic beliefs.

Consider the following strengthening of axiom $\mathrm{S} 2$ :

S2* (Binary Act Independence) For every essential $A \in \Sigma$, every $B \in\left\{A, A^{c}\right\}$, and for all $f, g, h \in \mathcal{F}$ such that $f=x A y, g=x^{\prime} A y^{\prime}$ and $h=x^{\prime \prime} A y^{\prime \prime}$. We have

$$
x A y \succcurlyeq x^{\prime} A y^{\prime} \Longrightarrow\left[c_{\left(x B x^{\prime \prime}\right)} A c_{\left(y B y^{\prime \prime}\right)}\right] \succcurlyeq\left[c_{\left(x^{\prime} B x^{\prime \prime}\right)} A c_{\left(y^{\prime} B y^{\prime \prime}\right)}\right]
$$

This is stronger than S2, as it removes all the conditions for its application, except that on the measurability of the acts. It is thus more in the spirit of the traditional independence axiom of EU. When substituted for S2, it gives a biseparable preference whose capacity is complement additive over all essential events. To obtain full complement additivity we only need to add an obvious necessary condition on preferences (beliefs, really):

S4 For all $A \in \Sigma$, if $A$ is null (resp. universal), then $A^{c}$ is non-null (resp. nonuniversal).

We now have the sought result:

Proposition 8 Suppose that $\succcurlyeq$ is an ambiguity averse (or loving) biseparable preference relation which satisfies $S 1$, and that $X$ is connected and separable. Then it satisfies axioms $S_{2 *}^{*}$ and $S_{4}$ if and only if $\rho$ is a probability measure. 
This proposition provides the axiomatic characterization of the model of biseparable preferences with probabilistic beliefs. To see that the latter is strictly weaker than SEU, recall the preferences in example (iv) in subsection 2.2: There $\rho=P$, but if $\beta>0$ the DM does not behave as a SEU maximizer (indeed, the larger $\beta$ is, the more ambiguity averse the DM's behavior is). The version of that model with variable $\beta(\cdot)$ also shows that having probabilistic beliefs is strictly weaker than being 'probabilistically sophisticated' in the sense of Machina and Schmeidler [21, who have a similar example]: Such $\succcurlyeq$ is probabilistically sophisticated only if the functional $N(\cdot)$ is increasing with respect to first-order stochastic dominance of the probability distributions that acts induce via $P$.

\section{$5 \quad$ Invariant Biseparable Preferences}

We now briefly consider a subset of the biseparable preferences which seems to be of some interest, even though it is strictly smaller than is needed for our characterization results. Recall that from Lemma 1 it follows that for any canonical representation $V$ of a biseparable preference relation $\succcurlyeq$, we can find a functional $I_{V}$ such that $V(f)=I_{V}(u(f))$ for all $f \in \mathcal{F}$. A natural question that this result poses is the following: Is the functional $I_{V}$ really dependent on the canonical representation? That is, if $V^{\prime}$ is another canonical representation of $\succcurlyeq$, is $I_{V^{\prime}}$ going to be equal to $I_{V}$ ? As we show presently, in general the anser to this question is: No. ${ }^{16}$ Thus, requiring that such uniqueness holds provides a smaller class of preferences, that we define as follows:

Definition 11 A biseparable preference relation $\succcurlyeq$ is invariant if there exists a monotone functional $I: B(\Sigma) \rightarrow \mathbb{R}$ such that $V(\cdot)=I(u(\cdot))$ for all canonical representations $V$ of $\succcurlyeq$. Such functional $I: B(\Sigma) \rightarrow \mathbb{R}$ is then called the canonical functional of $\succcurlyeq$.

By their definition and Proposition 3, invariant biseparable preferences feature uniqueness properties of all their basic components: $V$ is unique up to positive affine tranformations, $\rho$ is unique, and $I$ is unique.

The next simple result shows a property of the functional $I_{V}$ associated with a canonical representation $V$ that characterizes the invariant biseparable preference relations.

Proposition 9 A biseparable preference relation $\succcurlyeq$ is invariant if and only if there exists a canonical representation $V$ of $\succcurlyeq$ such that $I_{V}$ is constant-affine; that is,

$$
I_{V}(a \phi+b)=a I_{V}(\phi)+b
$$

for all $a>0, b \in \mathbb{R}$, and $\phi \in B(\Sigma)$. Moreover, such $I_{V}$ is the canonical functional of $\succcurlyeq$.

\footnotetext{
${ }^{16}$ However, by Proposition 3 the functional $I_{V}: B(\Sigma) \rightarrow \mathbb{R}$ is uniquely defined on binary functions of the form $u(f)$ : That is, given canonical representations $V$ and $V^{\prime}$ of $\succcurlyeq, I_{V}(\phi)=I_{V^{\prime}}(\phi)$ for all binary functions $\phi \in B(\Sigma)$ such that there are $f, f^{\prime} \in \mathcal{F}$ for which $u(f)=\phi=u^{\prime}\left(f^{\prime}\right)$.
} 
Hence, all the preferences presented in Subsection 2.2 are invariant if they are biseparable. In fact, all those preference functionals are seen to be constant-affine. The following example shows a non-invariant biseparable preference.

Example 1 Let $S$ be a finite state space, with $\Sigma=2^{S}$, and $X=[0,1]$ (with the Euclidean topology). Consider a preference defined as in the second part of example (iv) of subsection 2.2 , with utility $u^{*}$ equal to the identity, and $P: \Sigma \rightarrow \mathbb{R}$ a nondegenerate probability measure on $(S, \Sigma)$. The preference $\succcurlyeq$ is represented by the functional $V^{*}(f)=$ $I^{*}\left(u^{*}(f)\right)=I^{*}(f)$, with $I^{*}: B(\Sigma) \rightarrow \mathbb{R}$ defined as follows:

$$
I^{*}(\phi)=(1-\beta(\phi)) \int \phi d P+\beta(\phi) \varphi_{*}(\phi)
$$

where $\varphi^{*}$ is defined as in Eq. (6), and $\beta(\phi)=1 /(1+N(\phi))$ for a monotonic, supnorm continuous functional $N$ taking values in $[0,1]$. Such $\succcurlyeq$ is biseparable. In fact, the separability property on binary acts follows immediately by construction (with $\rho=$ $P)$. The monotonicity of $V^{*}$ is guaranteed by the separability and the monotonicity assumption on $N$. This shows that $V^{*}$ is a canonical representation of $\succcurlyeq$. Moreover, $\succcurlyeq$ is easily verified to satisfy S1. Hence, biseparability follows from Proposition 7.

However, the functional $I^{*}$ is not necessarily constant-affine. Suppose that $N$ satisfies the additional monotonicity condition: for any $b>0, \phi(s) \geq \psi(s)+b$ for all $s \in S$ implies $N(\phi)>N(\psi) \cdot{ }^{17}$ Then, when $a=1$ and $b>0$, for any $f$ it follows that $N\left(u^{*}(f)\right) \neq$ $N\left(a u^{*}(f)+b\right)$, so that we have

$$
I^{*}\left(a u^{*}(f)+b\right) \neq a I^{*}\left(u^{*}(f)\right)+b .
$$

This implies that $\succcurlyeq$ is not invariant biseparable. In fact, suppose there exists $I$ such that $V(f)=I(u(f))$ for all cardinal representations $V$ (and relative canonical utility $u$ ) of $\succcurlyeq$. Then, $V^{*}(f)=I\left(u^{*}(f)\right)$, so that, using $u^{*}(x)=x, I^{*}(\phi)=I(\phi)$ for all $\phi \in B(\Sigma)$ such that Range $(\phi) \subseteq[0,1]$. But this contradicts (13), because by Proposition $9, I$ has to be constant-affine.

While most of the well known examples of biseparable preferences are invariant, the results on risk attitude of Section 3 (as well as those on ambiguity attitude in [15]) do not require it. Since our focus here is on the most general preference model for which results of this sort (as well as the uniqueness of utility and beliefs) can be proved, we leave the axiomatic characterization of invariant biseparable preferences to future work. ${ }^{18}$

\footnotetext{
17 For an example of an $N$ with all the necessary requisites, consider $N(\phi)=\int_{S} e^{\phi(s)} Q(d s)$ for some nondegenerate probability measure $Q$ on $(S, \Sigma)$.

18 In view of Proposition 9, the characterization is straightforward in the Anscombe-Aumann setting: one only needs to strengthen axiom A2 to non-binary acts, thus obtaining Gilboa and Schmeidler's 'certainty independence' [18] axiom.
} 


\section{Appendix A Ceu and MEU Orderings}

A preference $\succcurlyeq$ is a $C E U$ ordering if there exist a utility index $u$ on $X$ and a capacity $\nu$ on $(S, \Sigma)$ such that, for all $f, g \in \mathcal{F}$,

$$
f \succcurlyeq g \Longleftrightarrow \int_{S} u(f(\cdot)) d \nu \geq \int_{S} u(g(\cdot)) d \nu
$$

where the integrals are taken in the sense of Choquet (notice that they are finite because each act in $\mathcal{F}$ is finite-valued). $\succcurlyeq$ is a $S E U$ ordering in the special case in which $\nu$ is a probability measure. An axiomatization of CEU (and SEU) preferences in the AnscombeAumann setting is found in Schmeidler [28]; one in the Savage setting is found, e.g., in Gilboa [17] and Chew and Karni [7].

Suppose that $C$ is a (weak*-) compact and convex set of probability measures on $(S, \Sigma)$. For a given real-valued $\Sigma$-measurable function $\varphi$, define $I_{C}(\varphi)$ as follows

$$
I_{C}(\varphi)=\min _{P \in C} \int_{S} \varphi(s) P(d s)
$$

Clearly, the operator $I_{C}$ is monotonic and positively homogenous. As for its additivity, say that two functions $\varphi, \psi: S \rightarrow \mathbb{R}$ are affinely related if either of the following holds: (i) there exist $\alpha>0$ and $\beta \in \mathbb{R}$ such that $\varphi(s)=\alpha \psi(s)+\beta$ for all $s \in S$, (ii) either function is constant. Ghirardato, Klibanoff and Marinacci [14] show that for every compact and convex $C, I_{C}$ is affine additive: If $\varphi, \psi: S \rightarrow \mathbb{R}$ are affinely related, then $I_{C}(\varphi+\psi)=$ $I_{C}(\varphi)+I_{C}(\psi)$. Thus, affine-relatedness is the counterpart of comonotonicity for the functionals of the type $I_{C}$. We remark that if two functions are affinely related, they are comonotonic, but the converse is generally false.

$\succcurlyeq$ is a $M E U$ ordering if there exist a utility index $u$ and a unique non-empty, compact and convex set $C$ of probabilities on $(S, \Sigma)$, such that, for all $f, g \in \mathcal{F}$,

$$
f \succcurlyeq g \Longleftrightarrow \min _{P \in C} \int_{S} u(f(\cdot)) d P \geq \min _{P \in C} \int_{S} u(g(\cdot)) d P .
$$

$\left(I_{C}[u(f(\cdot))]\right.$ is finite for every $f \in \mathcal{F}$, since $\mathcal{F}$ only contains simple acts.) As above, SEU corresponds to the special case of MEU in which $C=\{P\}$ for some probability measure $P$. An axiomatization of MEU preferences in the Anscombe-Aumann setting is in Gilboa and Schmeidler [18]; one in the Savage setting is in Casadesus-Masanell et al. [4].

\section{Appendix B Retrieving $V$ from given $u$ and $\rho$}

Here we characterize the set of the canonical representations which are compatible with a given utility function $u$ and a capacity $\rho$, under the assumption that the preference 
relation is biseparable. To provide such a characterization we need a piece of notation: given $\rho: \Sigma \rightarrow[0,1]$, define the functionals $\varphi^{*}: B(\Sigma) \rightarrow \mathbb{R}$ and $\varphi_{*}: B(\Sigma) \rightarrow \mathbb{R}$ as follows:

$$
\begin{aligned}
& \varphi_{*}(\phi)=\sup \left\{\int \phi^{\prime} d \rho: \phi^{\prime} \in B(\Sigma) \text { is two-valued, and } \phi^{\prime} \leq \phi\right\} \\
& \varphi^{*}(\phi)=\inf \left\{\int \phi^{\prime} d \rho: \phi^{\prime} \in B(\Sigma) \text { is two-valued, and } \phi^{\prime} \geq \phi\right\} .
\end{aligned}
$$

The integrals $\int \phi^{\prime} d \rho$ are Choquet integrals, which are well defined since $\rho$ is a capacity. Notice that $\varphi_{*}(\phi)=-\varphi^{*}(-\phi)$ for all $\phi \in B(\Sigma)$.

Proposition 10 A canonical representation $V$ of a biseparable preference relation $\succcurlyeq$ induces the pair $(u, \rho)$ if and only if $V(f)=I_{V}(u(f))$ for some monotone functional $I_{V}: B(\Sigma) \rightarrow \mathbb{R}$ such that, for all $\phi \in B(\Sigma)$,

$$
\varphi_{*}(\phi) \leq I_{V}(\phi) \leq \varphi^{*}(\phi) .
$$

Proof: Suppose $V: \mathcal{F} \rightarrow \mathbb{R}$ is such that $V(f)=I_{V}(u(f))$ for some monotone $I_{V}$ : $B(\Sigma) \rightarrow \mathbb{R}$ satisfying (16). For a binary act $f=x A y$, with $x \succ y$, we have $I_{V}(u(f))=$ $\varphi_{*}(u(f))=\varphi^{*}(u(f))$, and so

$$
V(x A y)=I_{V}(u(f))=\int u(f) d \rho=u(x) \rho(A)+u(y)(1-\rho(A)) .
$$

Conversely, suppose that $V$ is a canonical representation of $\succcurlyeq$ inducing $(u, \rho)$. Since $I_{V}$ is monotone, given any $\phi \in B(\Sigma)$, for all two-valued $\phi^{\prime} \in B(\Sigma)$ such that $\phi^{\prime} \geq \phi$ we have $\varphi^{*}\left(\phi^{\prime}\right)=I_{V}\left(\phi^{\prime}\right) \geq I_{V}(\phi)$, and so $\varphi^{*}(\phi)=\inf \varphi^{*}\left(\phi^{\prime}\right) \geq I_{V}(\phi)$. A similar argument shows that $\varphi_{*}(\phi) \leq I_{V}(\phi)$. Hence, $I_{V}$ satisfies (16).

\section{Appendix C Proofs}

This appendix contains the proof of all the results in the main text of the paper. For expositional reasons that will become clear as we proceed, the proofs are not presented in the same order as the statements in the paper. Precisely, we prove the main representation results of Section 4 (Theorems 3 and 4) before all the other results, which are then proved in the order in which they appear in the paper.

\section{C.1 Proofs of The Representation Theorems}

\section{C.1.1 Theorem 3}

We start by proving two lemmas, in which $\succcurlyeq$ is a preference relation satisfying B1-B3, A1-A3. 
Lemma 2 There exists $V: \mathcal{F} \rightarrow \mathbb{R}$, unique up to a positive affine transformation, such that for all $f, g \in \mathcal{F}$,

$$
f \succcurlyeq g \text { if and only if } V(f) \geq V(g)
$$

and for all binary $f \in \mathcal{F}, x \in X$, and $\alpha \in(0,1)$,

$$
V(\alpha f+(1-\alpha) x)=\alpha V(f)+(1-\alpha) V(x) .
$$

Proof: By axioms B1 and A2-A3 and the von Neumann-Morgenstern theorem, there exists $u: X \rightarrow \mathbb{R}$, unique up to positive affine transformations, such that for every $x, y \in X$, and every $\alpha \in[0,1]$,

$$
u(\alpha x+(1-\alpha) y)=\alpha u(x)+(1-\alpha) u(y)
$$

Given an act $f \in \mathcal{F}$, let $c_{f} \in X$ be one of its certainty equivalents (which exist by axiom A1). Define $V: \mathcal{F} \rightarrow \mathbb{R}$ by $V(f)=u\left(c_{f}\right)$. By A2, $f \sim c_{f} \in X$ implies $\alpha f+(1-\alpha) x \sim$ $\alpha c_{f}+(1-\alpha) x$, for all $x \in X$ and all binary $f \in \mathcal{F}$. Then, for all binary $f \in \mathcal{F}$ and $x \in X$,

$$
\begin{aligned}
V(\alpha f+(1-\alpha) x) & =V\left(\alpha c_{f}+(1-\alpha) x\right) \\
& =\alpha V(f)+(1-\alpha) V(x) .
\end{aligned}
$$

Moreover, let $V^{\prime}$ be another representation satisfying (17). Since $u^{\prime}$ is a positive affine transformation of $u$ there exist $\alpha>0$ and $\beta \in \mathbb{R}$ such that:

$$
\begin{aligned}
V^{\prime}(f) & =u^{\prime}\left(c_{f}\right)=\alpha u\left(c_{f}\right)+\beta \\
& =\alpha V(f)+\beta,
\end{aligned}
$$

as wanted.

Lemma 3 Let $V$ be the functional provided by Lemma 2. Then, for every $x^{*} \succ x_{*}$ and essential $A \in \Sigma$, if we normalize $V$ so that $V\left(x^{*}\right)=1, V\left(x_{*}\right)=0$, we have for all $x, y \in X$ such that $x \succcurlyeq y$,

$$
V(x A y)=V(y)+(V(x)-V(y)) V\left(x^{*} A x_{*}\right) .
$$

Proof: First notice that B2 and B3 imply that for all essential events $A \in \Sigma$, and all $x, y, z \in X$ such that $x, y \succcurlyeq z$,

$$
x \succcurlyeq y \Longleftrightarrow x A z \succcurlyeq y A z .
$$

or all $x, y, z \in X$ such that $x, y \preccurlyeq z$,

$$
x \succcurlyeq y \Longleftrightarrow z A x \succcurlyeq z A y .
$$

We first consider the case $x^{*} \succcurlyeq x \succ y \succcurlyeq x_{*}$. Let $\alpha \in(0,1]$ and $\beta \in[0,1)$ be such that

$$
x \sim \alpha x^{*}+(1-\alpha) y \text { and } y \sim \beta x^{*}+(1-\beta) x_{*} .
$$


W.l.o.g., set $V\left(x_{*}\right)=0$ and $V\left(x^{*}\right)=1$. Hence, $V(y)=\beta$, while $V(x)=\alpha+(1-\alpha) V(y)$ implies that

$$
V(x)-V(y)=\alpha[1-V(y)]=\alpha(1-\beta) .
$$

Therefore, using (17) and (18) we obtain:

$$
\begin{aligned}
V(x A y) & =V\left(\left(\alpha x^{*}+(1-\alpha) y\right) A y\right)=V\left(\alpha\left(x^{*} A y\right)+(1-\alpha) y\right) \\
& =\alpha V\left(x^{*} A y\right)+(1-\alpha) V(y) \\
& =\alpha V\left(x^{*} A\left(\beta x^{*}+(1-\beta) x_{*}\right)\right)+(1-\alpha) V(y) \\
& =\alpha V\left(\beta x^{*}+(1-\beta)\left(x^{*} A x_{*}\right)\right)+(1-\alpha) V(y) \\
& =\alpha\left[\beta V\left(x^{*}\right)+(1-\beta) V\left(x^{*} A x_{*}\right)\right]+(1-\alpha) V(y) \\
& =\alpha \beta V\left(x^{*}\right)+\alpha(1-\beta) V\left(x^{*} A x_{*}\right)+(1-\alpha) V(y) \\
& =\alpha V(y)+\alpha(1-\beta) V\left(x^{*} A x_{*}\right)+(1-\alpha) V(y) \\
& =V(y)+\alpha(1-\beta) V\left(x^{*} A x_{*}\right) \\
& =V(y)+(V(x)-V(y)) V\left(x^{*} A x_{*}\right)
\end{aligned}
$$

as wanted. To conclude the proof, we have to show the result in the remaining three cases: (i) $x \succcurlyeq x^{*}, y \succcurlyeq x_{*}$; (ii) $x^{*} \succ x, x_{*} \succ y$; (iii) $x \succ x^{*}, x_{*} \succ y$. As the proof for each case is analogous to the one above, we omit it.

Proof of Theorem 3: The $(i i) \Rightarrow(i)$ part is immediate. As to the $(i) \Rightarrow(i i)$ part, let $V$ be the functional provided by Lemma 2. Fix $x^{*} \succ x_{*}$ and, taking if necessary a positive affine transformation of $V$, suppose that $V\left(x^{*}\right)=1$ and $V\left(x_{*}\right)=0$. For all essential $A \in \Sigma$ set $\rho(A)=V\left(x^{*} A x_{*}\right)$, and for all null (resp. universal) $A \in \Sigma$, set $\rho(A)=0$ (resp. $\rho(A)=1$ ). By Lemma 3 and the definitions of null and universal, we then have $V(x A y)=u(x) \rho(A)+u(y)(1-\rho(A))$, so that $V$ and $\rho$ are the required functions.

As for the uniqueness statement, it is clear that any positive affine transformation of $V$ satisfies the representation with the $\rho$ constructed above. Suppose that $V^{\prime}$ is another representation and $\rho^{\prime}$ is another capacity which represent $\succcurlyeq$. From Lemma 2 it follows that $V^{\prime}$ must be a positive affine transformation of $V$. This fact allows us to show that $\rho(A)=\rho^{\prime}(A)$ for all $A \in \Sigma$ along the same lines as the proof of Proposition 3. Thus, $\rho$ is unique and $V$ is unique up to a positive affine transformation.

\section{C.1.2 Theorem 4}

The proof of Theorem 4 involves several lemmas. We start with a simple solvability result, whose proof is given for completeness. Given any $A \in \Sigma, x \in X$ and $g \in \mathcal{F}, x A g$ denotes the act such that $(x A g)(s)=x$ if $s \in A$, and $(x A g)(s)=g(s)$ if $s \notin A$.

Lemma 4 Let $\succcurlyeq$ be a preference relation satisfying B1, B2 and $S 1$. If $X$ is connected, then 
(a) for every $f \in \mathcal{F}$ there exists $x \in X$ such that $f \sim x$.

(b) for every $x, z \in X, A \in \Sigma$, and $f, g \in \mathcal{F}$, if $x A f \succ g \succ y A f$, there exists $x^{\prime} \in X$ such that $x^{\prime}$ Af $\sim g$.

Proof: We want to show that the set $\{x \in X: x A f \succcurlyeq g\}$ is $\tau$-closed in $X$ for all $f, g \in \mathcal{F}$. Let $\left\{x_{\alpha}\right\}_{\alpha \in D} \subseteq\{x \in X: x A f \succcurlyeq g\}$ be a net such that $x_{\alpha} \stackrel{\tau}{\longrightarrow} x_{0}$. Then $x_{\alpha} A f$ converges pointwise to $x_{0} A f$, and all acts are measurable to the same partition. By S1, if $x_{\alpha} A f \succcurlyeq g$ for all $\alpha \in D$, then $x_{0} A f \succcurlyeq g$. Hence, $x_{0} \in\{x \in X: x A f \succcurlyeq g\}$, which is therefore a $\tau$-closed set in $X$. In particular, if $A$ is universal, this implies that the set $\{x \in X: x \succcurlyeq g\}$ is $\tau$-closed in $X$. Symmetric arguments show that both sets $\{x \in X: x A f \preccurlyeq g\}$ and $\{x \in X: x \preccurlyeq g\}$ are $\tau$-closed in $X$. Both $(a)$ and $(b)$ now follow from the connectedness of $X$.

Presenting the next lemma requires introducing some terminology and notation. Given $m^{\prime \prime} \succ m^{\prime}$ and an essential event $A \in \Sigma$, we define an increasing standard sequence with mesh $\left(m^{\prime}, m^{\prime \prime}\right)$ and carrier $A$ a sequence $\left\{x^{0}, x^{1}, x^{2}, \ldots\right\} \subseteq X$ such that $x^{0} \succ m^{\prime \prime}$ and for every $n \geq 0, x^{n} A m^{\prime} \sim x^{n+1} A m^{\prime \prime}$ for all $n$ such that $x^{n}$ is not the last element of the sequence. Symmetrically, we define a emphdecreasing standard sequence with mesh $\left(m^{\prime}, m^{\prime \prime}\right)$ and carrier $A$ a sequence $\left\{x^{0}, x^{1}, x^{2}, \ldots\right\} \subseteq X$ such that $m^{\prime} \succ x^{0}$ and for every $n \geq 0, m^{\prime \prime} A x^{n+1} \sim m^{\prime} A x^{n}$ for all $n$ such that $x^{n}$ is not the last element of the sequence. Finally, we say that $\left\{x^{0}, x^{1}, x^{2}, \ldots\right\}$ is a standard sequence if there are a mesh and a carrier which make it into an increasing or decreasing standard sequence with respect to that mesh and carrier. A standard sequence $\left\{x^{0}, x^{1}, x^{2}, \ldots\right\}$ is said to be strictly bounded if there are $x_{*}, x^{*} \in X$ such that $x^{*} \succ x_{n} \succ x_{*}$ for every $n \geq 0$.

We can now state and prove:

Lemma 5 Let $\succcurlyeq$ be a preference relation satisfying B1-B3, and S1. If $X$ is a connected and separable topological space, every strictly bounded standard sequence is finite.

Proof: Since $X$ is connected and separable, there exists a continuous representation $\phi: X \rightarrow \mathbb{R}$ of the restriction of $\succcurlyeq$ to $X$. Moreover, B2 and B3 imply that for all essential events $A \in \Sigma$, and all $x, y, z \in X$ such that $x, y \succcurlyeq z$,

$$
x \succcurlyeq y \Longleftrightarrow x A z \succcurlyeq y A z .
$$

Define $V: \mathcal{F} \rightarrow \mathbb{R}$ by $V(f) \equiv \phi\left(c_{f}\right)$, where $c_{f}$ is the certainty equivalent of $f$. Clearly, $V(x)=\phi(x)$ for all $x \in X$. Let $x, y \in X$ be such that $x \succ y \succcurlyeq z$, and let $\alpha \in \mathbb{R}$ be such that $V(x A z)>\alpha>V(x A z)$. Let $c^{\prime} \sim x A z$ and $c^{\prime \prime} \sim y A z$. Then $\phi\left(c^{\prime}\right)>\alpha>\phi\left(c^{\prime \prime}\right)$. Being $\phi$ continuous, $\phi(X)$ is an interval, and so there is $c \in X$ such that $\phi(c)=\alpha$. Hence, $x A z \succ c \succ y A z$, and so, by Lemma 4, there is $x^{\prime} \in X$ such that $x^{\prime} A z \sim c$, which implies $V\left(x^{\prime} A z\right)=\alpha$.

Next, let $\left\{x_{n}\right\}_{n \geq 1}$ be a decreasing sequence with $x_{n} \succcurlyeq x_{n+1} \succcurlyeq z$ for all $n \geq 1$. If $\lim _{n \rightarrow \infty} \phi\left(x_{n}\right)=\phi(x)$, then $\lim _{n \rightarrow \infty} V\left(x_{n} A z\right)=V(x A z)$. In fact, suppose per contra 
that $\lim _{n \rightarrow \infty} V\left(x_{n} A z\right)=\alpha>V(x A z)$. By what we proved above, there exists $x^{\prime} \in X$ such that $V\left(x^{\prime} A z\right)=\alpha$, so that $V\left(x_{n} A z\right) \geq V\left(x^{\prime} A z\right)>V(x A z)$ for all $n \geq 1$. By (20), this implies that $x_{n} \succcurlyeq x^{\prime} \succ x$, which contradicts $\lim _{n \rightarrow \infty} \phi\left(x_{n}\right)=\phi(x)$. We conclude that $\lim _{n \rightarrow \infty} V\left(x_{n} A z\right)=V(x A z)$.

Let $\left\{x^{n}\right\}_{n \geq 0}$ be an increasing standard sequence with the essential event $A$ as carrier and mesh $\left(m^{\prime}, m^{\prime \prime}\right)$. By definition, $x^{n} \succ m^{\prime \prime} \succ m^{\prime}$ for all $n \geq 0$. Suppose $\left\{x^{n}\right\}_{n \geq 0}$ is strictly bounded by $x_{*}, x^{*} \in X$, i.e., $x^{*} \succ x^{n} \succ x_{*}$ for all $n \geq 0$. For all $n \geq 0$, we have

$$
\begin{aligned}
V\left(x^{n} A m^{\prime \prime}\right) & =V\left(x^{n+1} A m^{\prime}\right) \in[\phi(y), \phi(x)], \\
\phi\left(x^{n}\right) & \in\left[\phi\left(x_{*}\right), \phi\left(x^{*}\right)\right] .
\end{aligned}
$$

Since $\left[\phi\left(x_{*}\right), \phi\left(x^{*}\right)\right]$ is compact in $\mathbb{R}$, w.l.o.g. (taking subsequences if needed) we can assume that the sequence $\phi\left(x^{n}\right)$ converges to some $\alpha \in\left[\phi\left(x_{*}\right), \phi\left(x^{*}\right)\right]$. Since $\phi$ is continuous, $\left[\phi\left(x_{*}\right), \phi\left(x^{*}\right)\right]=\left\{x \in X: x_{*} \preccurlyeq x \preccurlyeq x^{*}\right\}$. Hence, there is $x^{\prime} \in X$ such that $\phi\left(x^{\prime}\right)=\alpha$, and so $\lim _{n \rightarrow \infty} \phi\left(x^{n}\right)=\phi\left(x^{\prime}\right)$. By what we proved above, if we take respectively $z=m^{\prime}$ and $z=m^{\prime \prime}$, we get $\lim _{n \rightarrow \infty} V\left(x^{n} A m^{\prime}\right)=V\left(x^{\prime} A m^{\prime}\right)$ and $\lim _{n \rightarrow \infty} V\left(x^{n} A m^{\prime \prime}\right)=V\left(x^{\prime} A m^{\prime \prime}\right)$. Since $V\left(x^{\prime} A m^{\prime}\right)<V\left(x^{\prime} A m^{\prime \prime}\right)$, this contradicts $V\left(x^{n} A m^{\prime \prime}\right)=V\left(x^{n+1} A m^{\prime}\right)$ for $n$ large enough. A symmetric argument holds for decreasing standard sequences.

Next, we provide two useful results on the representation of a preference relation satisfying axioms B1-B3, S1 and S2 when there are essential events. The first result shows that such a preference has a 'locally canonical' representation, holding for every act which is measurable w. r. t. the algebra generated by an essential event. Henceforth, for any $A \in \Sigma$ we let $\mathcal{F}_{A}$ be the set of (binary) acts which are measurable with respect to the algebra $\Sigma_{A}$ generated by $A$.

Lemma 6 Let $\succcurlyeq$ be a preference relation satisfying axioms B1-B3, S1, S2 with some essential event. Then for any essential $A \in \Sigma$, there is a representation $V_{A}: \mathcal{F} \rightarrow \mathbb{R}$ of $\succcurlyeq$ which satisfies: There exist a unique capacity $\rho_{A}: \Sigma_{A} \rightarrow[0,1]$ and a $\tau$-continuous function $u_{A}: X \rightarrow \mathbb{R}$, unique up to a positive affine transformation, such that, for all $x \succcurlyeq y$ and all $B \in \Sigma_{A}$,

$$
V_{A}(x B y)=u_{A}(x) \rho_{A}(B)+u_{A}(y)\left(1-\rho_{A}(B)\right) .
$$

Notice that the properties of $\rho_{A}$ and $u_{A}$ and (21) imply that $V_{A}(x)=u_{A}(x)$ for all $x \in X$, and that $V_{A}$ is unique only up to positive affine transformations. Also, $\rho_{A}(A) \in(0,1)$.

Proof: We first show that for any essential $A$, all hypotheses of Theorem 1 of Chew and Karni $\left[7\right.$, henceforth CK] are satisfied on the restriction $\succcurlyeq$ to the acts in $\mathcal{F}_{A}$. CK's axioms 1 ' and 5' are obviously satisfied. Their axiom 3 ' is also satisfied: It is easy to see that an event $A$ is 'null' in the CK-sense if and only if $A^{c}$ is universal, while $A$ is 'universal' in the $\mathrm{CK}$-sense if and only if $A^{c}$ is null. Hence, our eventwise monotonicity axiom is equivalent to their axiom 3'. Also, if $A$ is essential, then $A^{c} \in \Sigma_{A}$ is neither null nor universal in 
the CK-sense. Axioms 2 and 6 are satisfied by Lemma 4. Axiom 7 by Lemma 5. We can thus conclude that all of CK's axioms are satisfied.

By Theorem 1 of CK, there is a unique $\rho_{A}$ on $\Sigma_{A}$ and a $\tau$-continuous function $u_{A}$ : $X \rightarrow \mathbb{R}$, unique up to positive affine transformations, such that the functional $V_{A}: \mathcal{F}_{A} \rightarrow$ $\mathbb{R}$ defined by (21) represents $\succcurlyeq$ on $\mathcal{F}_{A}$. We extend $V_{A}$ to all of $\mathcal{F}$ by letting $V_{A}(f)=u_{A}\left(c_{f}\right)$ for any $f \in \mathcal{F}$. It is immediate to check that, thus defined, $V_{A}$ represents $\succcurlyeq$.

Lemma 7 Let $\succcurlyeq$ satisfy the assumptions of Lemma 6, and for any essential $A \in \Sigma$, let $V_{A}: \mathcal{F} \rightarrow \mathbb{R}$ be the representation obtained there. There exists a representation $V$ of $\succcurlyeq$, with a $\tau$-continuous restriction $u$ to $X$, and a capacity $\rho: \Sigma \rightarrow[0,1]$ such that, for all $A \in \Sigma$ and all consequences $x \succ y$,

$$
V(x A y)=u(x) \rho(A)+u(y)(1-\rho(A))
$$

if and only if for all essential $A \in \Sigma$, under a common normalization, $V_{A}(f)=V(f)$ for all $f \in \mathcal{F}$.

Proof: 'If' part: Given a common normalization of the $V_{A}$ 's, suppose that there exists $V: \mathcal{F} \rightarrow \mathbb{R}$ such that $V_{A}(f)=V(f)$ for all $f \in \mathcal{F}$ and all essential $A \in \Sigma$. Clearly, $V$ represents $\succcurlyeq$. Define $u: X \rightarrow \mathbb{R}$ by $u(x)=u_{A}(x)$ for all $x \in X$, and $\rho: \Sigma \rightarrow[0,1]$ by $\rho(B)=\rho_{A}(B)$ for all $B \in \Sigma_{A}$ and all essential $A \in \Sigma .^{19}$ If $A$ is null, set $\rho(A)=0$, while if $A$ is universal, set $\rho(A)=1$. Thus, for all $B \in \Sigma$ such that $B \in \Sigma_{A}$ for an essential $A \in \Sigma$ and all $x \succcurlyeq y$,

$V(x B y)=V_{A}(x B y)=u_{A}(x) \rho_{A}(B)+u_{A}(y)\left(1-\rho_{A}(B)\right)=u(x) \rho(B)+u(y)(1-\rho(B))$.

If, instead $B$ does not belong to any such $\Sigma_{A}$, it is easy to check that (3) holds by construction. This proves the 'if' part.

'Only if' part: Suppose that $\succcurlyeq$ admits $V$ and $\rho$ satisfying (22), and let $A \in \Sigma$ be an essential event. We can follow the steps of the proof of Lemma 6 to show that we can apply Theorem 1 of $\mathrm{CK}$ to $\succcurlyeq_{A}$, the restriction of $\succcurlyeq_{\text {to }} \mathcal{F}_{A}$. Thus, $\succcurlyeq_{A}$ admits a CEU representation $V_{A}$ which is unique only up to positive affine transformations. Since also the restriction of $V$ to $\mathcal{F}_{A}$ is a CEU representation of $\succcurlyeq_{A}$, on $\mathcal{F}_{A}$ the functionals $V$ and $V_{A}$ are positive affine transformations of each other. Given $x^{*} \succ x_{*}$, if we impose the normalization $u\left(x_{*}\right)=u_{A}\left(x_{*}\right)=0$ and $u\left(x^{*}\right)=u_{A}\left(x^{*}\right)=1$, we thence have $V_{A}(f)=V(f)$ for all $f \in \mathcal{F}_{A}$. In turn, this implies $\rho(B)=\rho_{A}(B)$ for all $B \in \Sigma_{A}$ and $u(x)=u_{A}(x)$ for all $x \in X$. Finally, for every $f \in \mathcal{F}$,

$$
V_{A}(f)=u_{A}\left(c_{f}\right)=u\left(c_{f}\right)=V(f) .
$$

This shows that for all essential $A \in \Sigma, V_{A}=V$ after a common normalization.

${ }^{19}$ Notice that if $A$ and $A^{c}$ are both essential then $\rho_{A}=\rho_{A^{c}}$ and (with the common normalization) $u_{A}=u_{A^{c}}$. This is due to the uniqueness properties of the representation in Lemma 6 , and to the fact that it is constructed for the algebra $\Sigma_{A}$, rather than the single events $A$ or $A^{c}$. 
Proof of Theorem 4: We prove existence first. The 'only if' part is immediate. As to the 'if' part, fix an essential $B \in \Sigma$, and a representation $V_{B}$ obtained by Lemma 6 . Using axiom S3, it is straightforward to mimic our proof of Proposition 1 in [15] to show that for any other essential $A \in \Sigma, u_{A}=u_{B}$ under a common normalization. Hence $V_{A}=V_{B}$ for every essential $A$, so that by Lemma $7, V \equiv V_{B}$ is such that $V(x B y)=$ $u(x) \rho(B)+u(y)(1-\rho(B))$. The cardinality of $V$ follows immediately the cardinality of the representations $V_{A}$ of Lemma 6 and Lemma 7. The uniqueness of $\rho$ is then proved as we did in the proof of Theorem 3.

As for continuity, we already know that $u$ is $\tau$-continuous. It remains to prove that $V: \mathcal{F} \rightarrow \mathbb{R}$ is sub-continuous. Let $\left\{f_{\alpha}\right\} \subseteq \mathcal{F}$ be a net that pointwise converges to $f \in \mathcal{F}$ such that all $f_{\alpha}$ 's and $f$ are measurable w.r.t. the same partition. Consider first a monotone increasing subnet $\left\{f_{\beta}\right\} \subseteq\left\{f_{\alpha}\right\}$ such that $\lim _{\beta} V\left(f_{\beta}\right)=\varlimsup_{\overline{1}} V\left(f_{\alpha}\right)$. Being a subnet, we clearly have that $\left\{f_{\beta}\right\}$ has the same properties as $\left\{f_{\alpha}\right\}$, so that $\lim _{\beta} u\left(f_{\beta}(s)\right)=u(f(s))$ for all $s \in S$. Let $x^{*}, x_{*} \in X$ be such that $u\left(x^{*}\right)=\max _{s \in S} u(f(s))$ and $u\left(x_{*}\right)=\min _{s \in S} u(f(s))$. Since $f$ is finite valued, both $x_{*}$ and $x^{*}$ exist. Suppose first that there exists $x^{\prime} \in X$ such that $x^{\prime} \succ x^{*}$. Let $\varepsilon=\left(u\left(x^{\prime}\right)-u\left(x^{*}\right)\right) / 2$. Since $f$ is a finite valued act, we can denote its range as $\left\{x_{1}, \ldots, x_{N}\right\}$. There exists a finite partition $\left\{A_{i}\right\}_{i=1}^{N} \subseteq \Sigma$ of $S$ such that $s \in A_{i}$ implies $\lim _{\beta} u\left(f_{\beta}(s)\right)=u\left(x_{i}\right)$. For each $i \in\{1, \ldots, N\}$ there exists $\beta_{\varepsilon}^{i}$ such that $\beta \geq \beta_{\varepsilon}^{i}$ and $s \in A_{i}$ implies $\left|u\left(f_{\beta}(s)\right)-u\left(x_{i}\right)\right| \leq \varepsilon$. Letting $\beta_{\varepsilon}=\max _{1 \leq i \leq N} \beta_{\varepsilon}^{i}$, we have that $\left|u\left(f_{\beta}(s)\right)-u\left(x_{i}\right)\right| 1_{A_{i}} \leq \varepsilon$ for all $\beta \geq \beta_{\varepsilon}$. In particular, this implies that for all $\beta \geq \beta_{\varepsilon}$ we have $V\left(f_{\beta}\right)-u\left(x^{*}\right) \leq \varepsilon$, so that $V\left(f_{\beta}\right) \leq u\left(x^{\prime}\right)-\varepsilon$. In turn, this implies that $\lim _{\beta} V\left(f_{\beta}\right) \leq u\left(x^{\prime}\right)-\varepsilon$. Given $\beta \geq \beta_{\varepsilon}$, let $x_{\beta} \in X$ be such that $u\left(x_{\beta}\right)=\min _{s \in S} u\left(f_{\beta}(S)\right)$. Let us consider any such $x_{\beta}$. Clearly, $u\left(x_{\beta}\right) \leq V\left(f_{\beta}\right) \leq u\left(x^{\prime}\right)-\varepsilon<u\left(x^{\prime}\right)$, and so

$$
u\left(x_{\beta}\right) \leq \lim _{\beta} V\left(f_{\beta}\right)<u\left(x^{\prime}\right) .
$$

Since $X$ is connected and $u$ continuous, (23) implies that there exists $\widehat{x} \in X$ such that $u(\widehat{x})=\lim _{\beta} V\left(f_{\beta}\right)$. Since $f_{\beta}$ is a monotone increasing net, $f_{\beta} \preccurlyeq \widehat{x}$ for all $\beta$, so by S1 $f \preccurlyeq \widehat{x}$. Hence,

$$
V(f) \leq u(\widehat{x})=\lim _{\beta} V\left(f_{\beta}\right)=\varlimsup_{\alpha} V\left(f_{\alpha}\right) .
$$

Now, suppose that such $x^{\prime}$ does not exist, i.e., $x \preccurlyeq x^{*}$ for all $x \in X$. This implies $f_{\beta} \preccurlyeq x^{*}$ for all $\beta$, and so, by S1, $f \preccurlyeq x^{*}$. If $\lim _{\beta} V\left(f_{\beta}\right)=u\left(x^{*}\right)$, we again get $V(f) \leq \varlimsup_{\alpha} V\left(f_{\alpha}\right)$. If, in contrast, $\lim _{\beta} V\left(f_{\beta}\right)<u\left(x^{*}\right)$, we have $u\left(x_{\beta}\right) \leq \lim _{\beta} V\left(f_{\beta}\right)<u\left(x^{*}\right)$ for all $\beta$, and so

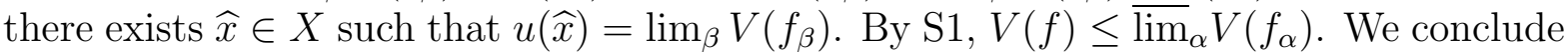
that $V(f) \leq \varlimsup_{\alpha} V\left(f_{\alpha}\right)$ holds in all cases.

Consider a monotone decreasing subnet $\left\{f_{\gamma}\right\} \subseteq\left\{f_{\alpha}\right\}$ such that $\lim _{\gamma} V\left(f_{\gamma}\right)=\underline{\lim }_{\alpha} V\left(f_{\alpha}\right)$. Again, being a subnet $\left\{f_{\gamma}\right\}$ shares the properties of $\left\{f_{\alpha}\right\}$, so that $\lim _{\gamma} u\left(f_{\gamma}(s)\right)=u(f(s))$ for all $s \in S$. Suppose first that there exists $x^{\prime \prime} \in X$ such that $x^{\prime \prime} \prec x_{*}$. Let $\varepsilon=\left(u\left(x_{*}\right)-u\left(x^{\prime \prime}\right)\right) / 2$. Proceeding as above, we can show that there exists $\gamma_{\varepsilon}$ such that for all $\gamma \geq \gamma_{\varepsilon}$ we have $u\left(x_{*}\right)-V\left(f_{\gamma}\right) \leq \varepsilon$, so that $V\left(f_{\gamma}\right) \geq u\left(x^{\prime \prime}\right)+\varepsilon$. This implies that $\lim _{\gamma} V\left(f_{\gamma}\right) \geq u\left(x^{\prime \prime}\right)+\varepsilon$. Given $\gamma \geq \gamma_{\varepsilon}$, let $x_{\gamma} \in X$ be such that $u\left(x_{\gamma}\right)=\max _{s \in S} u\left(f_{\gamma}(S)\right)$. 
Let us consider any such $x_{\gamma}$. Clearly, $u\left(x_{\gamma}\right) \geq V\left(f_{\gamma}\right) \geq u\left(x^{\prime \prime}\right)+\varepsilon>u\left(x^{\prime \prime}\right)$, and so

$$
u\left(x_{\gamma}\right) \geq \lim _{\gamma} V\left(f_{\gamma}\right)>u\left(x^{\prime \prime}\right) .
$$

Since $X$ is connected and $u$ continuous, (25) implies that there exists a $\widehat{x} \in X$ such that $=\lim _{\gamma} V\left(f_{\gamma}\right)$. Again, $f_{\gamma} \succcurlyeq \widehat{x}$ for all $\gamma$, and by S1 $f \succcurlyeq \widehat{x}$. Hence,

$$
V(f) \geq u(\widehat{x})=\lim _{\gamma} V\left(f_{\gamma}\right)=\varliminf_{\alpha} V\left(f_{\alpha}\right) .
$$

Now suppose that such a $x^{\prime \prime}$ does not exist, i.e., $x \succcurlyeq x_{*}$ for all $x \in X$. This implies $f_{\gamma} \succcurlyeq x_{*}$ for all $\gamma$, and so, by S1, $f \succcurlyeq x_{*}$. If $\lim _{\gamma} V\left(f_{\gamma}\right)=u\left(x_{*}\right)$, we get $V(f) \geq \underline{\lim }_{\alpha} V\left(f_{\alpha}\right)$. If $\lim _{\gamma} V\left(f_{\gamma}\right)>u\left(x_{*}\right)$, we have $u\left(x_{\gamma}\right) \geq \lim _{\gamma} V\left(f_{\gamma}\right)>u\left(x_{*}\right)$ for all $\gamma$, and so there exists $\widehat{x} \in X$ such that $u(\widehat{x})=\lim _{\gamma} V\left(f_{\gamma}\right)$. By S1, $V(f) \geq \underline{\lim }_{\alpha} V\left(f_{\alpha}\right)$ holds again. We conclude that $V(f) \geq \underline{\lim }_{\alpha} V\left(f_{\alpha}\right)$ holds in all cases.

Summing up, we have that

$$
\varliminf_{\alpha} V\left(f_{\alpha}\right) \leq V(f) \leq \varlimsup_{\alpha} V\left(f_{\alpha}\right) .
$$

To complete the proof, we have to show that $\varliminf_{\alpha} V\left(f_{\alpha}\right)=\varlimsup_{\alpha} V\left(f_{\alpha}\right)$. Suppose, per contra, that $\underline{\lim }_{\alpha} V\left(f_{\alpha}\right)<\varlimsup_{\alpha} V\left(f_{\alpha}\right)$. We showed that there exist $\widehat{x}_{1}, \widehat{x}_{2} \in X$ such that $u\left(\widehat{x}_{1}\right)=\underline{\lim }_{\alpha} V\left(f_{\alpha}\right)$ and $u\left(\widehat{x}_{2}\right)=\varlimsup_{\lim _{\alpha}} V\left(f_{\alpha}\right)$. Hence, being $X$ connected, there exist $x^{\prime}, x^{\prime \prime} \in X$ such that $\underline{\lim }_{\alpha} V\left(f_{\alpha}\right)<u\left(x^{\prime}\right)<u\left(x^{\prime \prime}\right)<\varlimsup_{\lim _{\alpha}} V\left(f_{\alpha}\right)$. Let

$$
\varepsilon=\min \left\{u\left(x^{\prime}\right)-u\left(\widehat{x}_{1}\right), \varlimsup_{\alpha} V\left(f_{\alpha}\right)-u\left(\widehat{x}_{2}\right)\right\} .
$$

There exist $\beta_{\varepsilon}$ and $\gamma_{\varepsilon}$ such that, for all $\beta \geq \beta_{\varepsilon}$ and all $\gamma \geq \gamma_{\varepsilon}$,

$$
u\left(\widehat{x}_{2}\right)-V\left(f_{\beta}\right) \leq \varepsilon \text { and } V\left(f_{\gamma}\right)-u\left(\widehat{x}_{1}\right) \leq \varepsilon,
$$

and so, for all $\beta \geq \beta_{\varepsilon}$ and all $\gamma \geq \gamma_{\varepsilon}$,

$$
V\left(f_{\beta}\right) \geq u\left(x^{\prime \prime}\right) \text { and } V\left(f_{\gamma}\right) \leq u\left(x^{\prime}\right) .
$$

By S1, this implies $V(f) \geq u\left(x^{\prime \prime}\right)$ and $V(f) \leq u\left(x^{\prime}\right)$, a contradiction. We conclude that $\underline{\lim }_{\alpha} V\left(f_{\alpha}\right)=\varlimsup_{\lim _{\alpha}} V\left(f_{\alpha}\right)$. That is, $V$ is sub-continuous.

\section{C.2 Proofs of the Other Results}

\section{C.2.1 Section 2}

Proof of Proposition 1: Let $V$ be a canonical representation of $\succcurlyeq$. We have $x \succcurlyeq y$ iff $x A x \succcurlyeq y A y$ iff $u(x) \geq u(y)$. Moreover, if $x \succ y$, we have

$$
\begin{aligned}
x A y \succcurlyeq x B y & \Longleftrightarrow V(x A y) \geq V(x B y) \\
& \Longleftrightarrow u(x) \rho_{V}(A)+u(y)\left(1-\rho_{V}(A)\right) \geq u(x) \rho_{V}(B)+u(y)\left(1-\rho_{V}(B)\right) \\
& \Longleftrightarrow[u(x)-u(y)] \rho_{V}(A) \geq[u(x)-u(y)] \rho_{V}(B) \\
& \Longleftrightarrow \rho_{V}(A) \geq \rho_{V}(B),
\end{aligned}
$$

as wanted. 
Proof of Proposition 2: Suppose that $A \in \Sigma$ is essential. By definition, there exist $x \succ y$ such that $x \succ x A y \succ y$. Hence, $u(x)>u(x) \rho_{V}(A)+u(y)\left[1-\rho_{V}(A)\right]>u(y)$, which implies $u(x)\left(1-\rho_{V}(A)\right)>u(y)\left(1-\rho_{V}(A)\right)$ and $u(x) \rho_{V}(A)>u(y) \rho_{V}(A)$. Hence, $\rho_{V}(A) \in$ $(0,1)$. As to the converse, $\rho_{V}(A) \in(0,1)$ implies, for all $x \succ y$,

$$
u(x)>u(x) \rho_{V}(A)+u(y)\left[1-\rho_{V}(A)\right]>u(y),
$$

and so $x \succ x A y \succ y$.

Next, suppose that $A$ is null. By definition, there exist $x, y \in X$ such that $x \succ y$ and $y \sim x A y$. Then, for all canonical representation $V$ of $\succcurlyeq$ we have $u(y)=u(x) \rho_{V}(A)+$ $u(y)\left[1-\rho_{V}(A)\right]$, and so $\rho_{V}(A)=0$ since $u(x) \neq u(y)$. The converse holds. In fact, suppose that $\rho_{V}(A)=0$. For any $x \succ y$ and any canonical representation $V$ we have $V(x A y)=u(y)$, and so $x A y \sim y$, which implies that $A$ is null. A similar argument shows that $\rho_{V}(A)=1$ iff $A$ is universal.

We conclude that $(i)$ and $(i i)$ hold. As to $(i i i)$, given any $x \succ y, A \subseteq B$ implies $x A y \leq x B y$, and so, being $V$ monotone,

$$
\rho_{V}(A)=\frac{V(x A y)-u(y)}{u(x)-u(y)} \leq \frac{V(x B y)-u(y)}{u(x)-u(y)}=\rho_{V}(B) .
$$

Proof of Proposition 3: Suppose that there exists some essential event. Then if $V$ and $V^{\prime}$ are two canonical representations of $\succcurlyeq$ there are $\alpha>0$ and $\beta \in \mathbb{R}$ such that $V=\alpha V^{\prime}+\beta$. Let $x \succ y$. By Eq. (3), for every essential $A \in \Sigma$,

$$
\rho_{V}(A)=\frac{V(x A y)-u(y)}{u(x)-u(y)}=\frac{(\alpha V(x A y)+\beta)-(\alpha u(y)+\beta)}{(\alpha u(x)+\beta)-(\alpha u(y)+\beta)}=\rho_{V^{\prime}}(A) .
$$

If $A \in \Sigma$ is not essential, then $\rho_{V}(A)=\rho_{V^{\prime}}(A)$ follows from part $(i i)$ of Proposition 2 .

\section{C.2.2 Section 3}

Proof of Theorem 1: We start by showing that $(i)$ implies $(i i)$. Ordinal equivalence follows immediately from the fact that $\succcurlyeq_{2}$ is more uncertainty averse than $\succcurlyeq_{1}$. As for the second part of the statement, let $x \succ y$ be any two consequences and take the common normalization $u_{1}(x)=u_{2}(x)=1$ and $u_{1}(y)=u_{2}(y)=0$. If $\rho_{1}(A) \in\{0,1\}$ for all $A \in \Sigma$, it is w.l.o.g. to assume that $u_{1}=u_{2}$, so there is nothing to prove. So assume that there is one $A \in \Sigma$ such that $0<\rho_{1}(A)=\rho_{2}(A)<1$ (equality of the $\rho_{i}$ 's follows from the assumption that the two preferences have a common canonical functional).

Consider the subset of $X$ thus defined:

$$
I \equiv\left\{z \in X: y \preccurlyeq_{1} z \preccurlyeq_{1} x\right\}=\left\{z \in X: y \preccurlyeq_{2} z \preccurlyeq_{2} x\right\}
$$


where the equality again follows from ordinal equivalence. We now construct a 'grid' of points on $I$, that we label $G$. Let $G_{0} \equiv\{y, x\}$. Find the certainty equivalent $c_{(x A y)}^{1}$ of $x A y$ for $\succcurlyeq_{1}$ and let $G_{1} \equiv\left\{y, c_{(x A y)}^{1}, x\right\}$. Inductively, define $G_{i} \equiv\left\{c_{\left(x^{\prime} A x^{\prime \prime}\right)}^{1}: x^{\prime}, x^{\prime \prime} \in G_{i-1}\right\}$ for $i=2,3, \ldots$, and let $G \equiv \lim _{i \rightarrow \infty} G_{i}=\cup_{i=0}^{\infty} G_{i}$ (notice that $G_{i-1} \subseteq G_{i}$ ).

We claim that $u_{2}(z) \geq u_{1}(z)$ for every $z \in G$. To prove this claim, we use induction on $i$. Consider $G_{1}$, and let $z=c_{(x A y)}^{1}$. By $(i)$ we have that

$$
z \sim_{1} x A y \Rightarrow z \succcurlyeq_{2} x A y
$$

which, in terms of the representations, is written $u_{2}(z) \geq \rho=u_{1}(z)$. Suppose now that the claim holds for every $z \in G_{j}$, for $j \leq i-1$. Consider $z \in G_{i}$. There are $x^{\prime}, x^{\prime \prime} \in G_{i-1}$ such that $z=c_{\left(x^{\prime} A x^{\prime \prime}\right)}^{1}$, and we know that $u_{2}\left(x^{\prime}\right) \geq u_{1}\left(x^{\prime}\right)$ and $u_{2}\left(x^{\prime \prime}\right) \geq u_{1}\left(x^{\prime \prime}\right)$. Hence,

$$
u_{2}\left(x^{\prime}\right) \rho+u_{2}\left(x^{\prime \prime}\right)(1-\rho) \geq u_{1}\left(x^{\prime}\right) \rho+u_{1}\left(x^{\prime \prime}\right)(1-\rho)=u_{1}(z) .
$$

Since from $(i)$ we also have $z \succcurlyeq_{2} x^{\prime} A x^{\prime \prime}$, we thus find $u_{2}(z) \geq u_{1}(z)$, as required. This concludes the induction step, and proves the claim.

Next, consider a $z \in I$. It is immediate to use the fact that $\rho \in(0,1)$ to show that $u_{1}(G)$ is a dense subset of the interval $\left[u_{1}(y), u_{1}(x)\right]$. Hence, there is a sequence $\left\{z_{n}\right\}_{n=1}^{\infty} \subseteq G$ for which $\lim _{n \rightarrow \infty} u_{1}\left(z_{n}\right)=u_{1}(z)$. We use ordinal equivalence to show that $\lim _{n \rightarrow \infty} u_{2}\left(z_{n}\right)=u_{2}(z)$ as well. Start by observing that it is w.l.o.g. to assume that either $u_{1}\left(z_{n}\right) \uparrow u_{1}(z)$ or $u_{1}\left(z_{n}\right) \downarrow u_{1}(z)$. By contradiction, suppose that $\lim _{n \rightarrow \infty} u_{2}\left(z_{n}\right)=$ $\alpha \neq u_{2}(z)$, in particular that $\alpha>u_{2}(z)$. If $u_{1}\left(z_{n}\right) \uparrow u_{1}(z)$, we immediately have a contradiction, since eventually $u_{2}\left(z_{n}\right)>u_{2}(z)$. So, suppose that $u_{1}\left(z_{n}\right) \downarrow u_{1}(z)$, implying $u_{2}\left(z_{n}\right)>u_{2}(z)$ for all $n$. Since $\rho \in(0,1)$, there is $N$ large enough so that

$$
u_{2}\left(z_{N}\right) \rho+u_{2}(z)(1-\rho)<\alpha .
$$

Hence, letting $f=z_{N} A z$, we have that $c_{f}$ is such that $z_{n} \succ_{2} c_{f}$ for every $n \geq N$ and $c_{f} \succ_{2} z$. On the other hand, $u_{1}\left(z_{N}\right)>u_{1}\left(c_{f}\right)>u_{1}(z)$, so that there is $N^{\prime}$ such that $u_{1}\left(z_{n}\right)<u_{1}\left(c_{f}\right)$ for $n \geq N^{\prime}$. Thus, for $n \geq \max \left[N, N^{\prime}\right]$ we have $z_{n} \succ_{2} c_{f}$ and $z_{n} \prec_{1} c_{f}$, a contradiction. The case in which $\alpha<u_{2}(z)$ is dealt with symmetrically.

This shows that for all $z \in I$, there is a sequence $\left\{z_{n}\right\} \subseteq G$ such that $u_{i}\left(z_{n}\right) \rightarrow u_{i}(z)$ for $i=1,2$. Since $u_{2}\left(z_{n}\right) \geq u_{1}\left(z_{n}\right)$ for all $n \geq 1$, (ii) now follows by continuity of the $u_{i}$ 's on $X$.

Next, we show that $(i i)$ implies $(i i i)$. Let $x, y \in X$ be as above. By ordinal equivalence, there exists an increasing function $\phi: \mathbb{R} \rightarrow \mathbb{R}$ such that $u_{2}(z)=\phi\left(u_{1}(z)\right)$ for all $z \in X$. We want to show that $\phi$ is concave. For each $\alpha \in(0,1)$ there exists a $z_{\alpha} \in X$ with $x \succ z \succ y$ and such that $u_{1}\left(z_{\alpha}\right)=\alpha u_{1}(x)+(1-\alpha) u_{1}(y)$. Then

$$
\begin{aligned}
\phi\left(\alpha u_{1}(x)+(1-\alpha) u_{1}(y)\right)=\phi\left(u_{1}\left(z_{\alpha}\right)\right) & =u_{2}\left(z_{\alpha}\right) \\
& \geq u_{1}\left(z_{\alpha}\right)=\alpha u_{2}(x)+(1-\alpha) u_{2}(y) \\
& =\alpha \phi\left(u_{1}(x)\right)+(1-\alpha) \phi\left(u_{1}(y)\right)
\end{aligned}
$$

and so $\phi$ is concave. The implication $(i i i) \Rightarrow(i i)$ is straightforward. 
Proof of Lemma 1: The 'if' part is obvious. As to the 'only if' part, let $f, g \in \mathcal{F}$ be such that $u(f(s))=u(g(s))$ for all $s \in S$. Then, $f(s) \sim g(s)$ for all $s \in S$, i.e., for all $s \in S$ we have both $f(s) \succcurlyeq g(s)$ and $f(s) \preccurlyeq g(s)$. By monotonicity, in turn this implies that $f \succcurlyeq g$ and $f \preccurlyeq g$, and so $f \sim g$. Since $V$ represents $\succcurlyeq$, this implies $V(f)=V(g)$. Hence, there exists $I^{\prime}: u(\mathcal{F}) \rightarrow \mathbb{R}$ such that $V(f)=I^{\prime}(u(f))$ for all $f \in \mathcal{F}$, where $u(\mathcal{F})=\{u(f): f \in \mathcal{F}\}$. We now show that $I^{\prime}$ is monotone on $u(\mathcal{F})$. Suppose that $f, g \in \mathcal{F}$ are such that $u(f(s)) \geq u(g(s))$ for all $s \in S$. Then, $f(s) \succcurlyeq g(s)$ for all $s \in S$, and so, by monotonicity, $V(f) \geq V(g)$, which proves that $I^{\prime}$ is monotone on $u(\mathcal{F})$. We now want to extend $I^{\prime}$ from $u(\mathcal{F})$ to $B(\Sigma)$.

Suppose that $u(X)$ is bounded below: there exists a positive integer $M$ such that $-M<u(x)$ for all $x \in X$. Set $m=\inf \left\{I^{\prime}(\psi): \psi \in u(\mathcal{F})\right\}$. Since all $\psi \in u(\mathcal{F})$ are finite-valued, we have $m \geq-M$. Given $\phi \in B(\Sigma)$, let $L_{\phi}=\{\psi \in u(\mathcal{F}): \psi \leq \phi\}$. Define $I: B(\Sigma) \rightarrow \mathbb{R}$ as follows:

$$
I(\phi)=\left\{\begin{array}{cc}
\sup \left\{I^{\prime}(\psi): \psi \in L_{\phi}\right\} & \text { if } L_{\phi} \neq \emptyset \\
m & \text { if } L_{\phi}=\emptyset
\end{array}\right.
$$

We show that $I(\phi) \in \mathbb{R}$ for all $\phi \in B(\Sigma)$. Suppose that $u(X)$ is bounded above, so that there exists a positive constant $M^{\prime}$ such that $M^{\prime}>u(x)$ for all $x \in X$. Since $u(\mathcal{F})$ consists of simple functions, for each $\psi \in u(\mathcal{F})$ there exists $x \in X$ such that $u(x) \geq \psi$. Hence, $M^{\prime}>\psi$ for all $\psi \in u(\mathcal{F})$, and so $I(\phi) \leq M^{\prime}$. Suppose that $u(X)$ is not bounded above. Let $\phi \in B(\Sigma)$. Since $\phi$ is finite-valued and $u(X)$ is unbounded, there exists $x \in X$ such that $u(x)>\phi$. Hence, $u(\mathcal{F}) \ni \psi \leq \phi$ implies $\psi<u(x)$, and so $I(\phi) \leq u(x)$. In both cases, $I(\phi) \in \mathbb{R}$. Let $\phi, \phi^{\prime} \in B(\Sigma)$ be such that $\phi \geq \phi^{\prime}$. Clearly, $L_{\phi^{\prime}} \subseteq L_{\phi}$. Suppose that $L_{\phi^{\prime}} \neq \emptyset$. Then $L_{\phi} \neq \emptyset$, and it is easy to check that $I(\phi) \geq I\left(\phi^{\prime}\right)$. Next suppose that $L_{\phi^{\prime}}=\emptyset$. By definition, $I\left(\phi^{\prime}\right)=m$. If $L_{\phi}=\emptyset, I(\phi)=m$ as well. If $L_{\phi} \neq \emptyset$, then there is some $\psi \in u(\mathcal{F})$ such that $I(\phi) \geq I^{\prime}(\psi) \geq m$. In both cases, $I(\phi) \geq I\left(\phi^{\prime}\right)$, thus showing that $I$ is monotone.

Suppose now that $u(X)$ is not bounded below. Then $L_{\phi} \neq \emptyset$ for all $\phi \in B(\Sigma)$. In fact, since $\phi$ is finite-valued, there exists $m^{\prime \prime}$ such that $m^{\prime \prime} \leq \phi$. But, for each such $m^{\prime \prime}$ there exists $x \in X$ with $u(x)<m^{\prime \prime}$, so that $u(x) \in L_{\phi}$. We can define $I: B(\Sigma) \rightarrow \mathbb{R}$ by $\sup \left\{I^{\prime}(\psi): \psi \in L_{\phi}\right\}$. Proceeding as above, such $I$ is shown to be a monotone functional extending $I^{\prime}$. This completes the proof of the 'only if' part.

Proof of Proposition 4: For $x \in X$ and $f \in \mathcal{F}$, suppose that $x \succcurlyeq_{1} f$. This implies $u_{1}(x) \geq I^{*}\left(u_{1}(f)\right)$, and so, being $\phi$ increasing, $\phi\left(u_{1}(x)\right) \geq \phi\left(I^{*}\left(u_{1}(f)\right)\right)$. But, by the Jensen property $(7)$, we have

$$
u_{2}(x)=\phi\left(u_{1}(x)\right) \geq \phi\left(I^{*}\left(u_{1}(f)\right)\right) \geq I^{*}\left(\phi\left(u_{1}(f)\right)\right)=I^{*}\left(u_{2}(f)\right),
$$

and so $x \succcurlyeq_{2} f$. A similar argument shows that $x \succ_{1} f \Longrightarrow x \succ_{2} f$. Now, observe that by the assumption on the range and those of Theorem 1, there are $x, y, x^{\prime}, y^{\prime} \in X$ such that $u_{1}(x)=u_{2}\left(x^{\prime}\right)$ and $u_{1}(y)=u_{2}\left(y^{\prime}\right)$. Hence, we can use the fact that $I^{*}$ is common to show that $\rho_{1}=\rho_{2}$. We can thus conclude that $\succcurlyeq_{2}$ is more risk averse than $\succcurlyeq_{1}$. 
Proof of Theorem 2: Suppose $\succcurlyeq$ exhibits preference for bet diversification. For every $z, z^{\prime} \in X$ and $\alpha \in[0,1]$, let $z \alpha z^{\prime} \equiv \alpha z+(1-\alpha) z^{\prime}$. We first show that for all $\alpha \in[0,1]$ and essential $A \in \Sigma,{ }^{20}$

$$
x A y \succcurlyeq x^{\prime} A y^{\prime} \Longrightarrow\left[x \alpha x^{\prime}\right] A\left[y \alpha y^{\prime}\right] \succcurlyeq x^{\prime} A y^{\prime} .
$$

Per contra, suppose that for some $\alpha^{*} \in[0,1]$ we have $x A y \succ x^{\prime} A y^{\prime}$ and $\left[x \alpha^{*} x^{\prime}\right] A\left[y \alpha^{*} y^{\prime}\right] \prec$ $x^{\prime} A y^{\prime}$. Let $V$ be the canonical representation of $\succcurlyeq$ whose utility index $u$ is continuous. For all $\alpha \in[0,1]$, set

$$
G(\alpha) \equiv V\left(\left[x \alpha x^{\prime}\right] A\left[y \alpha y^{\prime}\right]\right)=u\left(x \alpha x^{\prime}\right) \rho_{V}(A)+u\left(y \alpha y^{\prime}\right)\left(1-\rho_{V}(A)\right) .
$$

Since $u$ is continuous and $\rho_{V}(A) \in(0,1), G(\cdot)$ is continuous, so that $I \equiv\{G(\alpha): \alpha \in$ $\left.\left[\alpha^{*}, 1\right]\right\}$ is an interval. $I$ contains $V(x A y)$ (for $\left.\alpha=1\right)$ and $V\left(\left[x \alpha^{*} x^{\prime}\right] A\left[y \alpha^{*} y^{\prime}\right]\right)$, and also $V\left(x^{\prime} A y^{\prime}\right)$ by the assumption. Thus, there exists $\alpha^{\prime} \in\left[\alpha^{*}, 1\right]$ such that $G\left(\alpha^{\prime}\right)=V\left(x^{\prime} A y^{\prime}\right)$, so that $\left[x \alpha^{\prime} x^{\prime}\right] A\left[y \alpha^{\prime} y^{\prime}\right] \sim x^{\prime} A y^{\prime}$. Choose $\beta \in[0,1]$ such that $\alpha^{\prime} \beta=\alpha^{*}$. Then, by preference for bet diversification,

$$
\beta\left(\left[x \alpha^{\prime} x^{\prime}\right] A\left[y \alpha^{\prime} y^{\prime}\right]\right)+(1-\beta)\left(x^{\prime} A y^{\prime}\right) \succcurlyeq x^{\prime} A y^{\prime},
$$

which contradicts the assumption and proves (27).

Following [6], we now apply a result of Debreu [10]. Let $X^{o}=\left(x_{*}, x^{*}\right)$ be the interior of the interval $X$. For $\alpha \in X^{o}$, set $X^{\alpha} \equiv\left\{x \in X^{o}: x<\alpha\right\}$ and $X_{\alpha} \equiv\left\{x \in X^{o}: x>\alpha\right\}$. Define $F: X_{\alpha} \times X^{\alpha} \rightarrow \mathbb{R}$ by $F(x, y)=V(x A y)$. By (27), $F$ is quasi-concave. Moreover, $F$ is separable since $F(x, y)=u(x) \rho_{V}(A)+u(y)\left(1-\rho_{V}(A)\right)$. It follows that $u$ is concave on $X_{\alpha}$ or $X^{\alpha}$. Now, let

$$
X^{*} \equiv \bigcup_{\left\{\alpha \in X^{o}: u \text { is concave on } X^{\alpha}\right\}} X^{\alpha} \text { and } X_{*} \equiv \bigcup_{\left\{\alpha \in X^{o}: u \text { is concave on } X_{\alpha}\right\}} X_{\alpha} .
$$

Since for each $\alpha \in X^{o}, u$ is either concave on $X^{\alpha}$ or on $X_{\alpha}$, we have $X^{o} \subseteq X^{*} \cup X_{*}$. As $X^{o}$ is connected and $X^{*}$ and $X_{*}$ are open, $X^{*} \cap X_{*}$ is non-empty and open, so there are $z, z^{\prime} \in X^{*} \cap X_{*}$ with $z<z^{\prime}$. It follows that $u$ is concave on both $\left(x_{*}, z^{\prime}\right)$ and $\left(z, x^{*}\right)$. Hence, $D^{+} u$ is non-increasing on both $\left(x_{*}, z^{\prime}\right)$ and $\left(z, x^{*}\right)$, so that it is non-increasing on $X^{o}$. Since $u$ is continuous on $X^{o}$, this implies that $u$ is concave on $X^{o}$ (see, e.g., Royden [26, p. 114]). Being $u$ continuous on $X$, in turn this implies the concavity of $u$ on $X$.

As for the converse, suppose that $u$ is concave on $X$. For essential $A \in \Sigma$ and $x, x^{\prime}, y, y^{\prime} \in X$ such that $x \succ y$ and $x^{\prime} \succ y^{\prime}$, suppose that $x A y \sim x^{\prime} A y^{\prime}$. Then,

$$
\begin{aligned}
V\left(\left[x \alpha x^{\prime}\right] A\left[y \alpha y^{\prime}\right]\right)= & u\left(x \alpha x^{\prime}\right) \rho_{V}(A)+u\left(y \alpha y^{\prime}\right)\left(1-\rho_{V}(A)\right) \\
\geq & \alpha\left[u(x) \rho_{V}(A)+u(y)\left(1-\rho_{V}(A)\right)\right] \\
& +(1-\alpha)\left[u\left(x^{\prime}\right) \rho_{V}(A)+u\left(y^{\prime}\right)\left(1-\rho_{V}(A)\right)\right] \\
= & \alpha V(x A y)+(1-\alpha) V\left(x^{\prime} A y^{\prime}\right)=V\left(x^{\prime} A y^{\prime}\right),
\end{aligned}
$$

as wanted.

\footnotetext{
${ }^{20}$ For $u$ increasing, this is proved in Proposition 3.1 of Chateauneuf and Tallon [6].
} 
Proof of Proposition 5: $(i) \Rightarrow(i i)$ : Suppose that $u$ is concave on $X$, and set $\rho(A)=$ $\rho(B)=p \in(0,1)$. Since $p x+(1-p) y=p x^{\prime}+(1-p) y^{\prime}$, we have

$$
y^{\prime}-y=\frac{p}{1-p}\left(x-x^{\prime}\right) .
$$

We have $y^{\prime} \leq y \leq x \leq x^{\prime}$. Suppose that both $x^{\prime} \neq x$ and $y \neq y^{\prime}$. By concavity,

$$
\frac{u\left(x^{\prime}\right)-u(x)}{x^{\prime}-x} \leq \frac{u(y)-u\left(y^{\prime}\right)}{y-y^{\prime}}
$$

and so

$$
u\left(y^{\prime}\right) \leq u(y)+\frac{u\left(x^{\prime}\right)-u(x)}{x^{\prime}-x}\left(y^{\prime}-y\right) .
$$

Using (28) and (29), we can write:

$$
\begin{aligned}
p u\left(x^{\prime}\right)+(1-p) u\left(y^{\prime}\right) & \leq p u\left(x^{\prime}\right)+(1-p)\left[u(y)+\frac{u\left(x^{\prime}\right)-u(x)}{x^{\prime}-x}\left(y^{\prime}-y\right)\right] \\
& =p u\left(x^{\prime}\right)+(1-p) u(y)+(1-p) \frac{u\left(x^{\prime}\right)-u(x)}{x^{\prime}-x}\left(y^{\prime}-y\right) \\
& =p u\left(x^{\prime}\right)+(1-p) u(y)+(1-p) \frac{u\left(x^{\prime}\right)-u(x)}{x^{\prime}-x}\left(\frac{p}{1-p}\right)\left(x-x^{\prime}\right) \\
& =p u\left(x^{\prime}\right)+(1-p) u(y)+p\left(u(x)-u\left(x^{\prime}\right)\right) \\
& =p u(x)+(1-p) u(y)
\end{aligned}
$$

so that $x A y \succcurlyeq x^{\prime} B y^{\prime}$. If $x=x^{\prime}$, then $x A y \succcurlyeq x^{\prime} B y^{\prime}$ follows by B2. The same if $y=y^{\prime}$.

The $(i i) \Rightarrow(i i i)$ statement is immediate. As for the $(i i i) \Rightarrow(i)$ statement, we prove the contrapositive. Assume that $u$ is not concave. Then there are $x, y \in X$ and $\alpha \in(0,1)$ such that

$$
u(\alpha x+(1-\alpha) y)<\alpha u(x)+(1-\alpha) u(y) .
$$

W.l.o.g., assume that $u(x)>u(y)$. Since $\rho(\Sigma)=[0,1]$, there is $A \in \Sigma$ such that $\rho(A)=\alpha$. Consider the act $f=x A y$ and the constant act $E V(f)=\alpha x+(1-\alpha) y$. Then

$$
u(E V(f))=u(\alpha x+(1-\alpha) y)<\alpha u(x)+(1-\alpha) u(y)=V(f) .
$$

This proves that $\succcurlyeq$ has a preference for the subjective expected value.

\section{C.2.3 Section 4}

Proof of Proposition 6: Let $V$ be a functional representing $\succcurlyeq$, and let $\rho$ be defined as in the proposition's statement. For $f \in \mathcal{F}$, suppose that $f=\sum_{k=1}^{n} x_{k} 1_{A_{k}}$, with $x_{1} \succ x_{2} \succ$ $\cdots \succ x_{n}$ and $\left\{A_{1}, A_{2}, \ldots, A_{n}\right\}$ a measurable partition of $\Sigma$ (by axiom B2, it is w.l.o.g. to restrict our attention to this case). Define $B_{i}=\cup_{k=1}^{i} A_{k}$ and let $j$ be the smallest $i$ such that $B_{i}$ is universal. It is easy to check that $\int u(f) d \rho=V\left(x_{j}\right)$. We now show that $f \sim x_{j}$. 
Start by observing that by B2, if $A$ is universal, then $x \sim x A g$ for any $g$ such that $x \succcurlyeq g(s)$ for all $s \in S$, and $x \succ g(s)$ for some $s$. For, let $y \in X$ be the $(\succcurlyeq)$ minimal consequence that $g$ yields, so that $x \succ y$ by assumption. Since $A$ is universal, we have that

$$
x \sim x A y \preccurlyeq x A g \preccurlyeq x,
$$

where the second and third preferences follow from B2 and the definition of $y$. Hence $x \sim x A g$. Similarly, one shows that if $A$ is null, then $y \sim g A y$ for any $g$ such that $g(s) \succcurlyeq x$ for all $s \in S$, and $g(s) \succ x$ for some $s$.

Now, let $j$ be the smallest $i$ such that $B_{i}$ is universal, so that $B_{j-1}$ is null. By the fact we just showed, we have

$$
f B_{j-1} x_{j} \sim x_{j} \sim x_{j} B_{j} f
$$

It also follows from B2 and the choice of $j$ that

$$
f \preccurlyeq f B_{j-1} x_{j} \text { and } \quad x_{j} B_{j} f \preccurlyeq f
$$

from which we conclude that $f \sim x_{j}$, as wanted. Thus, every $V$ can be represented as in (9), which clearly implies that $V$ is canonical (monotonicity follows from the properties of Choquet integrals).

Proof of Proposition \%: Suppose that $\succcurlyeq$ is a preference with a canonical representation which satisfies $\mathrm{S} 1$. In the case in which $\succcurlyeq$ has no essential event, there is nothing to prove, so assume that there is an essential event $A$. It is easy to verify that since it has a canonical representation $V, \succcurlyeq$ satisfies axioms B1-B3 and S2-S3. Given that by assumption $\succcurlyeq$ also satisfies S1, we can then mimic the proof of the 'only if' part of Lemma 7 to show that $V=V_{A}$, one of the CEU representations on $\mathcal{F}_{A}$, after a common normalization. Since this is true for every canonical representation $V$, they are all normalizable positive affine transformations of each other. Hence $\succcurlyeq$ is biseparable.

The proof of Proposition 8 requires the following lemma, which is of some independent interest:

Lemma 8 Suppose that $X$ is connected and separable, and that $\succcurlyeq$ is a biseparable preference satisfying axiom $S 1$. Then $\succcurlyeq$ satisfies $S_{2}^{*}$ and $S_{4}$ if and only if $\rho$ is complement additive.

Proof: We prove the 'only if' first. As in the proof of Proposition 7 above, it is immediate to show that $\succcurlyeq$ satisfies axioms B1-B3 and S3. Given that it satisfies also axioms S1 and $\mathrm{S}^{*}$, we can now repeat the proof of Lemmas 6 to show that for every essential $A \in \Sigma$ there is $\rho_{A}$ and $u_{A}$ which represent the restriction of $\succcurlyeq$ to $\mathcal{F}_{A}$. However, it also follows from Theorem 1 of CK that $\rho_{A}$ is additive; that is, $\rho_{A}(A)+\rho_{A}\left(A^{c}\right)=1$. Since $\rho$ was defined as $\rho(A)=\rho_{A}(A)$ for every $A \in \Sigma$ (recall footnote 19), it follows that $\rho$ is complement additive on all essential events. The fact that $\rho$ is complement additive on the inessential 
events follows immediately from axiom $\mathrm{S} 4$ and Proposition 2. In fact, suppose that $A$ is null, so that $\rho(A)=0$. S4 implies that $A^{c}$ is non-null. If $A^{c}$ was essential, it would follow from the discussion above that $\rho(A)=1-\rho\left(A^{c}\right)>0$, a contradiction. Hence, $A^{c}$ must be universal, so that $\rho\left(A^{c}\right)=\rho(A)+\rho\left(A^{c}\right)=1$. The case of $A$ universal is proved similarly. This concludes the proof of the 'only if' part. The proof of the 'if' part is immediate.

Proof of Proposition 8: It follows from Lemma 8 that under the stated assumptions $\rho$ is complement additive iff $\mathrm{S}^{*}$ and $\mathrm{S} 4$ hold. From the discussion in the main text, we know that a biseparable preference which is ambiguity averse (resp. loving) has a willingness to bet which is dominated (resp. dominates) a probability. To conclude the proof we just have to observe (for the simple proof, see [16, Lemma 2]) that a capacity which is dominated or dominates a probability is complement additive iff it is additive.

\section{C.2.4 Section 5}

Proof of Proposition 9: 'Only if' part: Suppose that $I$ is the canonical functional of $\succcurlyeq$. Let $\phi \in u(\mathcal{F})$. By definition, there exists $f \in \mathcal{F}$ such that $\phi=u(f)$. Given any $a>0$ and $b \in \mathbb{R}$, let $V^{\prime}$ be such that $V^{\prime}=a V+b$. Then

$$
I(a \phi+b)=I\left(u^{\prime}(f)\right)=V^{\prime}(f)=a V(f)+b=a I(u(f))+b=a I(\phi)+b .
$$

Now, let $\phi \in B(\Sigma)$. Since $u(X)$ is an interval, there exist $\phi_{V} \in u(\mathcal{F}), a^{\prime}>0$, and $b^{\prime} \in \mathbb{R}$ such that $\phi=a^{\prime} \phi_{V}+b^{\prime}$. Then, for all $a>0$ and $b \in \mathbb{R}$,

$$
\begin{aligned}
I(a \phi+b) & =I\left(a\left(a^{\prime} \phi_{V}+b^{\prime}\right)+b\right)=I\left(a a^{\prime} \phi_{V}+a b^{\prime}+b\right) \\
& =a a^{\prime} I\left(\phi_{V}\right)+a b^{\prime}+b=a\left[a^{\prime} I\left(\phi_{V}\right)+b^{\prime}\right]+b \\
& =a I\left(a^{\prime} \phi_{V}+b^{\prime}\right)+b=a I(\phi)+b .
\end{aligned}
$$

'If' part: suppose there exists $I_{V}: B(\Sigma) \rightarrow \mathbb{R}$ such that

$$
I_{V}(a \phi+b)=a I_{V}(\phi)+b
$$

for all $\phi \in B(\Sigma), a>0$, and $b \in \mathbb{R}$. Let $B_{u}=\{a \psi+b: \psi \in u(\mathcal{F}), a>0, b \in \mathbb{R}\}$. For each $\phi \in B_{u}$, let $I(\phi)=a I_{V}(\psi)+b$, where $\phi=a \psi+b$ for some $\psi \in u(\mathcal{F}), a>0$, and $b \in \mathbb{R}$. We first show that $I$ is well defined on $B_{u}$. Suppose that there exist $\psi^{\prime} \in u(\mathcal{F}), a^{\prime}>0$, and $b^{\prime} \in \mathbb{R}$ such that $\phi=a^{\prime} \psi^{\prime}+b^{\prime}$. Simple algebra shows that $I_{V}(\psi)=\left(a^{\prime} / a\right) \psi^{\prime}+\left(b^{\prime}-b\right) / a$ because $I_{V}$ has property (30). Rearranging, we have $a I_{V}(\psi)+b=a^{\prime} I_{V}\left(\psi^{\prime}\right)+b^{\prime}$, and so $I$ is well defined.

Let $V^{\prime}$ be any another canonical representation of $\succcurlyeq$. There exist $a>0$ and $b \in \mathbb{R}$ such that $V^{\prime}=a V+b$. Hence, being $u^{\prime}(f) \in B_{u}$, we have:

$$
I\left(u^{\prime}(f)\right)=I(a u(f)+b)=a I_{V}(u(f))+b=a V(f)+b=V^{\prime}(f) .
$$




\section{References}

[1] Maurice Allais. Le comportement de l'homme rationnel devant le risque: Critique des postulats et axiomes de l'école américaine. Econometrica, 21:503-546, 1953.

[2] Frank J. Anscombe and Robert J. Aumann. A definition of subjective probability. Annals of Mathematical Statistics, 34:199-205, 1963.

[3] Kenneth J. Arrow. The theory of risk aversion. In Essays in the Theory of RiskBearing, chapter 3. North-Holland, Amsterdam, 1974. (Part of the Yriö Jahnssonin Säätio lectures in Helsinki, 1965).

[4] R. Casadesus-Masanell, P. Klibanoff, and E. Ozdenoren. Maxmin expected utility over savage acts with a set of priors. Journal of Economic Theory, forthcoming.

[5] Alain Chateauneuf. On the existence of a probability measure compatible with a total preorder on a boolean algebra. Journal of Mathematical Economics, 14:43-52, 1985.

[6] Alain Chateauneuf and Jean-Marc Tallon. Diversification, convex preferences and non-empty core. Mimeo, Université Paris I, July 1998.

[7] Soo Hong Chew and Edi Karni. Choquet expected utility with a finite state space: Commutativity and act-independence. Journal of Economic Theory, 62:469-479, 1994.

[8] Gustave Choquet. Theory of capacities. Annales de l'Institut Fourier (Grenoble), 5:131-295, 1953.

[9] Bruno de Finetti. Sulla preferibilità. Giornale degli Economisti e Annali di Economia, 6:3-27, 1952.

[10] Gerard Debreu and Tjalling Koopmans. Additively decomposed quasi-convex functions. Mathematical Programming, 24:1-38, 1982.

[11] Eddie Dekel. Asset demands without the independence axiom. Journal of Economic Theory, 57:163-169, 1989.

[12] Daniel Ellsberg. Risk, ambiguity, and the Savage axioms. Quarterly Journal of Economics, 75:643-669, 1961.

[13] Larry G. Epstein. A definition of uncertainty aversion. Review of Economic Studies, 66:579-608, 1999.

[14] Paolo Ghirardato, Peter K. Klibanoff, and Massimo Marinacci. Additivity with multiple priors. Journal of Mathematical Economics, 30:405-420, 1998.

[15] Paolo Ghirardato and Massimo Marinacci. Ambiguity made precise: A comparative foundation. Mimeo, Caltech and Università di Bologna (Revised: November 1999), October 1997. 
[16] Paolo Ghirardato and Massimo Marinacci. Range convexity and ambiguity averse preferences. Social Science Working Paper 1081, Caltech, January 2000.

[17] Itzhak Gilboa. Expected utility with purely subjective non-additive probabilities. Journal of Mathematical Economics, 16:65-88, 1987.

[18] Itzhak Gilboa and David Schmeidler. Maxmin expected utility with a non-unique prior. Journal of Mathematical Economics, 18:141-153, 1989.

[19] Faruk Gul. Savage's theorem with a finite number of states. Journal of Economic Theory, 57:99-110, 1992.

[20] David H. Krantz, R. Duncan Luce, Patrick Suppes, and Amos Tversky. Foundations of Measurement: Additive and Polynomial Representations, volume 1. Academic Press, San Diego, 1971.

[21] Mark J. Machina and David Schmeidler. A more robust definition of subjective probability. Econometrica, 60:745-780, 1992.

[22] Yutaka Nakamura. Subjective expected utility with non-additive probabilities on finite state spaces. Journal of Economic Theory, 51:346-366, 1990.

[23] Emre Ozdenoren. Auctions and bargaining with a set of priors. Mimeo, Northwestern University, January 2000.

[24] John W. Pratt. Risk aversion in the small and in the large. Econometrica, 32:122$136,1964$.

[25] John Quiggin. A theory of anticipated utility. Journal of Economic Behavior and Organization, 3:94-101, 1982.

[26] H. L. Royden. Real Analysis. Macmillan, New York, third edition, 1988.

[27] Leonard J. Savage. The Foundations of Statistics. Wiley, New York, 1954.

[28] David Schmeidler. Subjective probability and expected utility without additivity. Econometrica, 57:571-587, 1989.

[29] Amos Tversky and Daniel Kahneman. Advances in prospect theory: Cumulative representation of uncertainty. Journal of Risk and Uncertainty, 5:297-323, 1992.

[30] Peter P. Wakker. Additive Representations of Preferences. Kluwer, Dordrecht, 1989.

[31] Menachem E. Yaari. Some remarks on measures of risk aversion and on their uses. Journal of Economic Theory, 1:315-329, 1969.

[32] Menachem E. Yaari. The dual theory of choice under risk. Econometrica, 55:95-116, 1987. 